\title{
新型有机硫(硒)替加氟衍生物的合成及抗肿瘤活性研究
}

\author{
李宁波*,a,b,c 续 立 ${ }^{a}$ 马 榕 ${ }^{a}$ 范琪 ${ }^{a}$ 李 波 $b$ \\ 乔 洁 $a, b$ 郭崄*,a,c 许新华 $d$ \\ ( ${ }^{a}$ 山西医科大学生物化学与分子生物学教研室 山西太原 030001) \\ $\left({ }^{b}\right.$ 山西医科大学医用化学教研室 山西太原 030001) \\ ( ${ }^{c}$ 山西医科大学细胞生理学教育部重点实验室 山西太原 030001) \\ ( 湖南大学化学化工学院 长沙 410082)
}

\begin{abstract}
摘要 以替加氟为原料, 与氯代烷基醇反应生成中间体 $N$-羊仝烷基替加氟; 然后与对甲苯磺酰氯反应制得替加氟烷基磺 酸酯; 在双核钛全氟丁基磺酸配合物/锌粉催化体系下, 替加氟烷基磺酸酯与二芳基二硫(硒)梄反应, 较高产率得到一 系列新型芳基 $\left(N^{3}\right.$ - 替加氟烷基)硫(硒)醚衍生物. 其结构通过 ${ }^{1} \mathrm{H} N \mathrm{NM},{ }^{13} \mathrm{H}$ NMR 和 HRMS 确认. 对目标化合物进行了关 于结肠癌细胞 HCT116 和胃癌细胞 SGC-7901 的体外抗肿瘤活性测试. 结果表明, 绝大多数目标化合物比替加氟的抗肿 瘤活性高. DAPI (4',6-diamidino-2-phenylindole)对 HCT116 细胞染色实验及流式细胞仪定量检测实验表明，替加氟衍生 物可通过诱导细胞凋亡而抑制细胞生长. 此外, 有机硫(硒)替加氟衍生物作用于正常人胚胎肾细胞 HEK 293 的毒性比 替加氟低.
\end{abstract}

关键词＼cjkstart替加氟；二芳基二硫(硒)醚; 不对称硫(硒)醚；抗肿瘤活性；细胞凋亡

\section{Synthesis and Antitumor Activities of Novel Organic Sulfur (Selenium) Tegafur Derivatives}

\author{
$\mathrm{Li}$, Ningbo ${ }^{* a, b, c} \quad \mathrm{Xu}, \mathrm{Li}^{a} \quad \mathrm{Ma}, \mathrm{Rong}^{a} \quad \mathrm{Fan}^{a} \mathrm{Qi}^{a} \quad \mathrm{Li}, \mathrm{Bo}^{b}$ \\ Qiao, Jie ${ }^{a, b}$ Guo, Rui ${ }^{*, a, c} \quad \mathrm{Xu}, \mathrm{Xinhua}^{d}$ \\ ( ${ }^{a}$ Department of Biochemistry and Molecular Biology, Shanxi Medical University, Taiyuan, Shanxi 030001) \\ $\left({ }^{b}\right.$ Department of Chemistry, Shanxi Medical University, Taiyuan, Shanxi 030001) \\ $\left({ }^{c}\right.$ Key Laboratory of Cellular Physiology, Shanxi Medical University, Ministry of Education, Taiyuan, Shanxi 030001) \\ $\left({ }^{d}\right.$ College of Chemistry and Chemical Engineering, Hunan University, Changsha 410082)
}

\begin{abstract}
Tegafur, as a raw material, reacted with chloroalkyl alcohols to give the intermediate $N$-hydroxyalkyl tegafur, then which reacted with $p$-toluenesulfonyl chloride to obtain tegafluoroalkyl sulfonate. A series of novel aryl ( $N^{3}$-tegafluoroalkyl) thio- and seleno-ether derivatives were efficiently synthesized by the reaction of tegafluoroalkyl sulfonate with diaryl disulfides or diselenides under binuclear titanium(IV) salophen perfluorobutanesulfonate/zinc catalytic system. Their structures of the target products were confirmed by ${ }^{1} \mathrm{H}$ NMR, ${ }^{13} \mathrm{C}$ NMR and HRMS. Their antitumor activities were evaluated by colorectal carcinoma HCT116 cells and gastric carcinoma SGC-7901 cells. The preliminary bioassay results showed that most target compounds had more antitumor activities than tegafur. Moreover, HCT116 cells staining experiments (DAPI) and quantitative determination by flow cytometry indicated that the growth inhibition was associated with the induction of apoptosis. In addition, organic sulfur (selenium) tegafur derivatives had lower toxicity than tegafur on human embryonic kidney HEK 293 cells. Keywords tegafur; diaryl disulfides (diselenides); unsymmetrical sulfides (selenides); antitumor activity; apoptosis
\end{abstract}

替加氟又称呋喃氟尿嘧啶，是一种抗肿瘤药物．它＼cjkstart能够阻止肿瘤细胞内嘧啶类核苷酸的形成, 主要用于治

\footnotetext{
* Corresponding authors. E-mail: ningboli@sxmu.edu.cn; ruiguo@sxmu.edu.cn

Received December 28, 2020; revised March 30, 2021; published online April 16, 2021.

Project supported by the National Natural Science Foundation of China (No. 21802093), the Scientific and Technological Innovation Programs of Higher Education Institutions in Shanxi Province (No. 2019L0408), and the PhD Start-up Foundation of Shanxi Medical University (No. 03201501).

国家自然科学基金(No. 21802093)、山西省高等学校科技创新(No. 2019L0408)、山西医科大学博士启动基金(No. 03201501)资助项目.
} 
疗胃癌、结肠癌、直肠癌和胰腺癌等癌症 ${ }^{[1-5]}$. 但 5-氟尿 嘧啶类化合物脂溶性低, 选择性差, 且对中枢神经具有 一定的毒性 ${ }^{[6]}$. 目前, 国内外的一些研究学者对替加氟 的结构进行了修饰和改造 ${ }^{[7-13]}$. 如 2008 年, 高文远课题 组 ${ }^{[8]}$ 报道合成了含芳环酰亚胺结构的替加氟衍生物 (Scheme 1a), 并进行了体外抗肿瘤测试. 2012 年, 尹述 凡课题组 ${ }^{[9]}$ 报道了替加氟分子上引入亲脂性的茄尼醇, 从而改善化合物的脂溶性(Scheme 1b), 进一步研究了 目标化合物的抗肿瘤活性. 2015 年, 刘景平课题组 ${ }^{[12]}$ 报 道合成了含异羟肜酸或 2-氨基苯甲酰胺替加氟前体药 物(Scheme 1c), 并揭示了该药物对结肠癌细胞 HCT116 的抗癌机制. 然而, 上述修饰的替加氟药物中, 部分药 物仍然存在药性低、选择性差以及毒性大等问题 ${ }^{[14]}$. 因 此, 如何增强替加氟药物抗肿瘤活性, 同时减少毒副作 用成为众多学者关注的焦点.
有机硫化物及含硫的药物分子具有抗肿瘤、增强免疫力 和治疗心脑血管疾病等作用 ${ }^{[15-18]}$. 硒作为机体必需的微 量元素之一，同样具有重要的生物活性. 同时，有机硒 药物因具有优良的抗氧化、抗肿瘤、抗炎及免疫调节等 作用成为研究的热点 ${ }^{[19-23]}$. 替加氟 3 号位 $\mathrm{N}$ 连接的氢原 子具有部分酸性, 因此设想在替加氟 3 号位 $\mathrm{N}$ 原子上引 入烷基，增加药物脂溶性，再引入含硫或硒的基团，使 有机硫或硒基团和替加氟药物协同发挥作用，抗肿瘤活 性叠加 ${ }^{[24-25]}$. 报道合成了 25 种新型含硫或硒替加氟衍 生物, 并对目标化合物进行了体外抗肿瘤活性测试.

\section{1 结果与讨论}

如 Scheme 2 合成路线所示, 首先替加氟(1)与氯代 烷基醇在碱性条件下反应，得到中间体 $N$-羟烷基替加

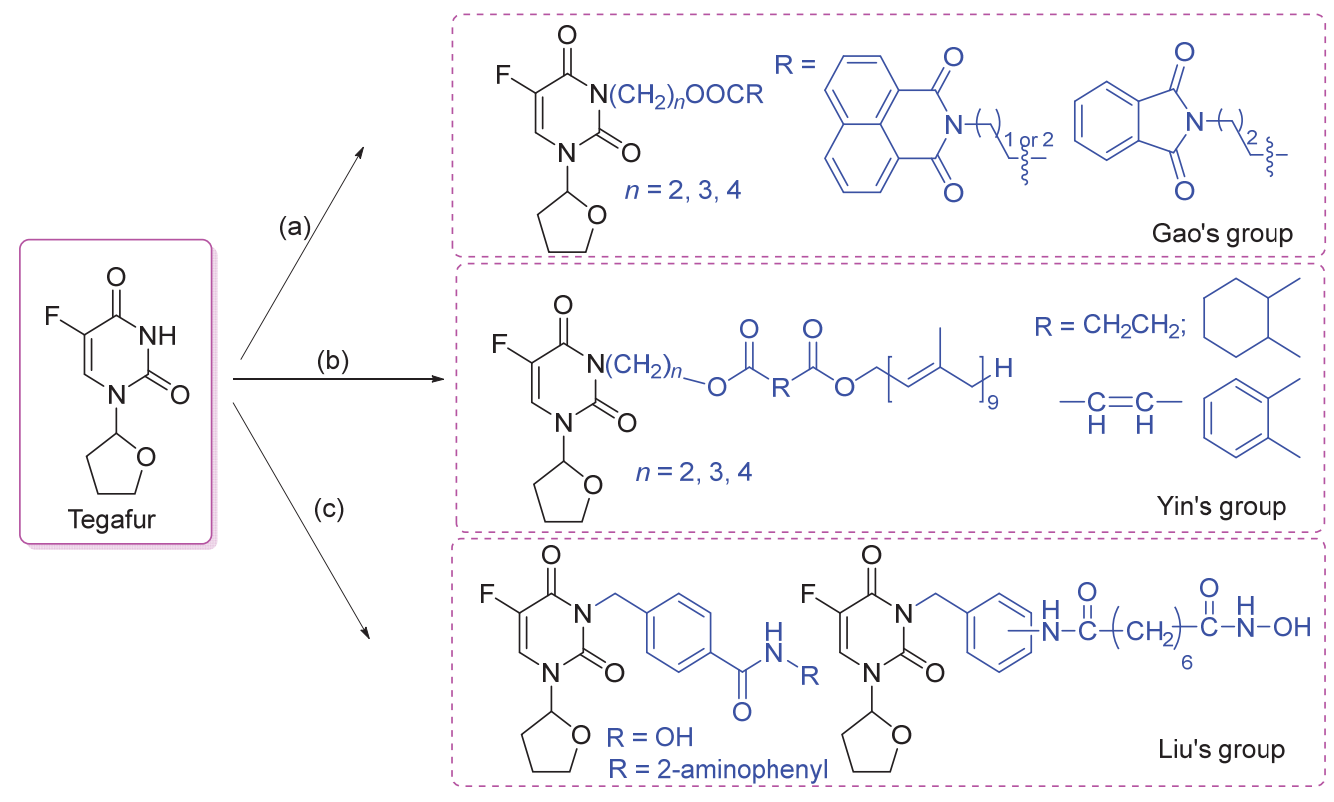

图式 $1 N^{3}$ 修饰的替加氟衍生物的合成

Scheme 1 Synthesis of $N^{3}$-modified tegafur derivatives

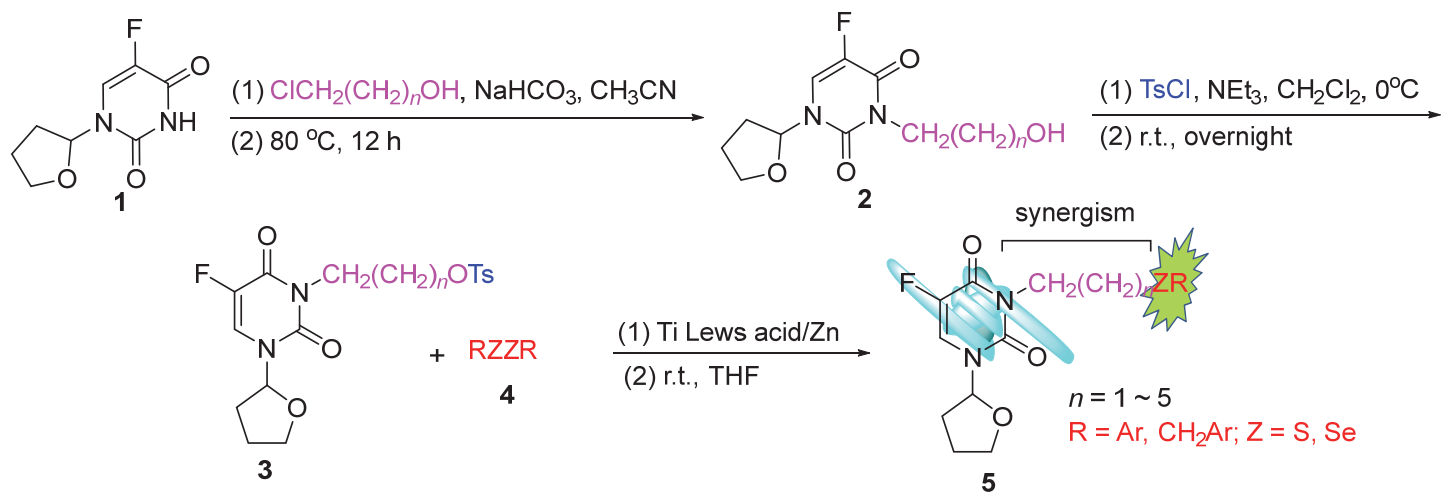

图式 2 芳基 $\left(N^{3}\right.$-替加氟烷基)硫(硒)醚衍生物的合成

Scheme 2 Synthesis of aryl ( $N^{3}$-tegafluoroalkyl)thio- and seleno-ethers derivatives 
氟(2); 接着与对甲苯磺酰氯反应得到替加氟烷基磺酸 酯(3). 我们前期研究表明, 双核钛全氟丁基磺酸配合 物/锌粉催化体系能够很好地催化还原断裂二芳基二硫 (硒)醚, 与卤代烷发生反应制得不对称硫(硒)梄[26]. 因 此, 在双核钛全氟丁基磺酸配合物/锌粉催化体系下, 通 过替加氟烷基磺酸酯(3)与二芳基二硫(硒)醚反应, 制备 得到新型的芳基 ( $N^{3}$-替加氟)烷基硫(硒)醚衍生物 $\mathbf{5}$.

以替加氟正丙基磺酸酯 $(3 \mathrm{~b}, 1 \mathrm{mmol}$ )和二苯基二硫 醚 $(\mathbf{4 a}, 0.5 \mathrm{mmol})$ 作为模型反应, 探究有机钛路易斯酸催 化剂剂量、锌粉剂量、溶剂和时间对反应的影响, 结果 见表 1. 如表 1 所示, 缺少有机钠全氟丁基磺酸催化剂 和锌粉任意一种, 反应均不会发生(Entries 1 2). 前期 研究表明, 有机钛配合物/锌粉体系能够高效催化还原 断裂二硫醚 ${ }^{[26]}$. 因此, 在两者同时存在的情况下, 反应 能够顺利进行(Entry 3). 当钛催化剂为 $5 \mathrm{~mol} \%$, 锌粉为 $1.2 \mathrm{mmol}$ 时, 反应产率达到最高(Entry 4); 继续增加钛 催化剂或锌粉的量, 产率几乎不变(Entries 5,6). 当反应 时间缩短至 $1 \mathrm{~h}$, 产率仍高达 $94 \%$ (Entry 7); 但继续缩短 至 $0.5 \mathrm{~h}$, 产率降低(Entry 8). 进一步探讨了溶剂对反应 的影响，使用 $\mathrm{CH}_{3} \mathrm{CN} 、 \mathrm{CH}_{2} \mathrm{Cl}_{2} 、 N, N$-二甲基甲酰胺(DMF) 和甲苯作溶剂时, 产率一般(Entries 9 12). 因此, 最佳 反应条件: 在氮气保护下, 钛催化剂 $5 \mathrm{~mol} \%$, 锌粉为 $1.2 \mathrm{mmol}$, 四氢呋喃(THF)为溶剂, 室温下反应 $1 \mathrm{~h}$.

表 1 催化剂剂量、温度、时间和溶剂对反应的影响 ${ }^{a}$ Table 1 Influence of catalyst dosage, temperature, time and solvent to the reaction

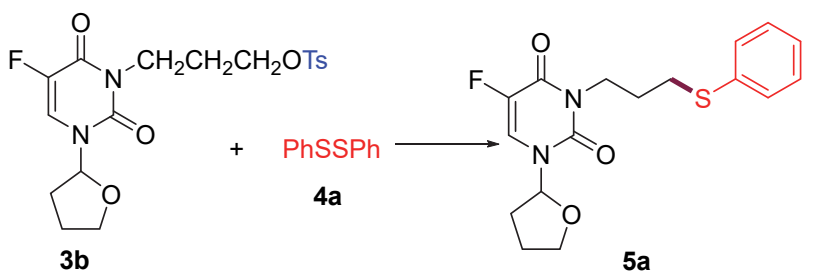

\begin{tabular}{cccccc}
\hline Entry & Cat./mol\% $\%$ & $\begin{array}{c}\text { Zn dust/ } \\
\text { mmol }\end{array}$ & Solvent & Time/h & Yield $^{b} \%$ \\
\hline 1 & 5 & 0 & THF & 12 & 0 \\
2 & 0 & 1 & THF & 12 & 0 \\
3 & 5 & 1 & THF & 4 & 82 \\
4 & 5 & 1.2 & THF & 4 & 94 \\
5 & 7 & 1.2 & THF & 4 & 93 \\
6 & 5 & 1.5 & THF & 4 & 94 \\
6 & 5 & 1.2 & THF & 1 & 94 \\
7 & 5 & 1.2 & THF & 0.5 & 73 \\
8 & 5 & 1.2 & $\mathrm{CH}_{3} \mathrm{CN}$ & 1 & 72 \\
9 & 5 & 1.2 & $\mathrm{CH}_{2} \mathrm{Cl} 2$ & 1 & 47 \\
10 & 5 & 1.2 & DMF & 1 & 68 \\
11 & 5 & 1.2 & Toluene $_{2}$ & 1 & 26 \\
\hline
\end{tabular}

${ }^{a}$ Tegafluoropropyl sulfonate (3b): $1 \mathrm{mmol}$; diphenyl disulfide (4a): $0.5 \mathrm{mmol}$; solvent: $3 \mathrm{~mL}$; r.t.; $\mathrm{N}_{2} ;{ }^{b}$ isolated yield.
在最优条件下，系统考察了替加氟烷基磺酸酯与二 芳基二硫(硒)醚的反应, 结果见表 2. 表 2 结果可知, 有 机钣全氟丁基磺酸催化剂/锌粉体系能够很好催化替加 氟正丙基磺酸酯与二芳基二硫(硒)醚反应得到芳基 $\left(N^{3}\right.$ 替加氟)烷基硫(硒)醚衍生物. 对于二芳基二硫醚而言, 苯环上的取代基对反应产率有一定的影响. 苯环上连有 吸电子基团 $\left(\mathrm{F}, \mathrm{Cl}, \mathrm{NO}_{2}\right)$ 比连有供电子基团 $\left(\mathrm{CH}_{3}, \mathrm{OH}\right.$, $\mathrm{NH}_{2}$ ) 的二芳基二硫醚活性略高，它们容易被还原断裂 生成芳硫基负离子, 从而进攻替加氟正丙基磺酸酯, 较 高产率得到目标产物 $\mathbf{5 b} \sim \mathbf{5 i}$. 苯环上硝基取代基的位置 对二芳基二硫醚活性也有一定的影响，对位产物产率比 邻位和间位略高 $(\mathbf{5 d} \sim \mathbf{5 f})$. 含有酰胺基团的二硫醚也显 示了很好的活性, 产物产率为 $90 \%$ (5j). 还考察了含杂 环的二硫醚化合物(三唑基、2-糠基、2-噻吩基及 2-吡啶 基)与替加氟正丙基磺酸酯的反应，产率为 $83 \% \sim 93 \%$ $(5 k \sim 5 n)$. 另外, 替加氟 3 号位 $\mathrm{N}$ 原子上连接的烷基链 的长短对产物产率影响不大, 随着碳链逐渐延长, 产物 产率略微降低 $(5 \mathrm{a}, 5 \mathrm{5o} \sim 5 \mathrm{r})$. 侧链为正己基的替加氟磺 酸酯也能够与三唑基二硫醚反应, 生成对应的目标化合 物, 产率为 91\% (5s). 最后, 探究了二芳基二硒醚与替 加氟正丙基磺酸酯反应. 结果显示, 含不同取代基的二 苯基二硒醚 $\left(\mathrm{CH}_{3}, \mathrm{OCH}_{3}, \mathrm{CN}, \mathrm{Cl}\right)$ 均能够生成对应目标化 合物, 而苯环上连有吸电子基团的二苯基二硒醚显示了 较高的活性 $(\mathbf{5 u} \sim \mathbf{5 x})$. 此外，二苄基二硒醚也能够与替 加氟正丙基磺酸酯反应, 产物产率为 $81 \%$ (5y).

采用 CCK-8 法测试了替加氟及 25 个芳基 $\left(N^{3}\right.$-替加 氟烷基)硫(硒)醚衍生物对结肠癌细胞 HCT116 和胃癌细 胞 SGC-7901 的体外抗肿瘤活性. 结果表明, 有机硫(硒) 替加氟衍生物对两种癌细胞均具有杀伤作用. 随着样品 浓度增加, 癌细胞死亡率升高. 测试了替加氟衍生物对 两种癌细胞的半抑制浓度 $\mathrm{IC}_{50}$, 结果见表 3. 研究结果 可知, 替加氟对 HCT116 和 SGC-7901 细胞的 $\mathrm{IC}_{50}$ 值分 别为 $861.13 \mu \mathrm{mol} / \mathrm{L}$ 和 $1000.42 \mu \mathrm{mol} / \mathrm{L}$. 除化合物 $\mathbf{5 e}, \mathbf{5 h}$ 和 50 的 $\mathrm{IC}_{50}$ 值比替加氟的略高外, 绝大多数化合物对 两种癌细胞的抑制作用比替加氟强. 对于有机硫替加氟 衍生物而言, 苯环上的取代基及取代基位置对两种癌细 胞抑制作用均有影响. 苯环含卤素 $(\mathrm{F}, \mathrm{Cl})$ 替加氟衍生物 比苯环无取代基的化合物 $\mathbf{5 a}$ 对癌细胞抑制活性高 $(\mathbf{5 a}$ 5c). 苯环上对位和邻位硝基取代的替加氟衍生物远高 于间位硝基取代的衍生物对两种癌细胞抑制活性 (5d 5f). 含供电子基团 $\left(\mathrm{CH}_{3}, \mathrm{OH}, \mathrm{NH}_{2}\right)$ 的苯硫基替加氟衍生 物也显示了较好的抑制活性 $(\mathbf{5 g} \sim \mathbf{5 i})$. 但对羟基苯硫基 替加氟衍生物 $5 \mathbf{h}$ 对 HCT116 的抑制活性低于替加氟. 苯 环含酰胺基团的替加氟衍生物 $5 \mathrm{i}$ 同样能够较好的抑制 两种癌细胞增殖, 对 SGC-7901 细胞的抑制作用更加明 
表 2 ( $N^{3}$-替加氟烷基)硫(硒)醚衍生物的合成

Table 2 Synthesis of aryl ( $N^{3}$-tegafluoroalkyl) thio- and seleno-ethers derivatives ${ }^{a, b}$

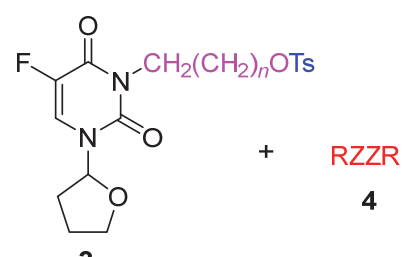

(1) Ti Lews acid/Zn

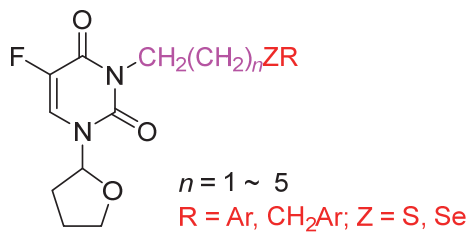

5<smiles>O=c1c(F)cn(C2CCCO2)c(=O)n1CCCSc1ccccc1</smiles>

5a, $94 \%$<smiles>O=c1c(F)cn(C2CCCO2)c(=O)n1CCCSc1ccccc1F</smiles>

5b, $92 \%$<smiles>O=c1c(F)cn(C2CCCO2)c(=O)n1CCCSc1ccc(I)cc1</smiles>

5c, $95 \%$<smiles>O=c1c(F)cn(C2CCCO2)c(=O)n1CCCSc1ccc([N+](=O)[O-])cc1</smiles>

5d, $94 \%$<smiles>O=c1c(F)cn(C2CCCO2)c(=O)n1CCCSc1cccc([N+](=O)[O-])c1</smiles>

5e, $92 \%$<smiles>O=c1c(F)cn(C2CCCO2)c(=O)n1CCCSc1ccccc1[N+](=O)[O-]</smiles>

5f, $89 \%$<smiles>Cc1ccc(SCCCn2c(=O)c(F)cn(C3CCCO3)c2=O)cc1</smiles>

$5 \mathrm{~g}, 88 \%$<smiles>O=c1c(F)cn(C2CCCO2)c(=O)n1CCCSc1ccc(O)cc1</smiles>

5h, $85 \%$<smiles>Nc1ccc(SCCCn2c(=O)c(F)cn(C3CCCO3)c2=O)cc1</smiles>

5i, $84 \%$

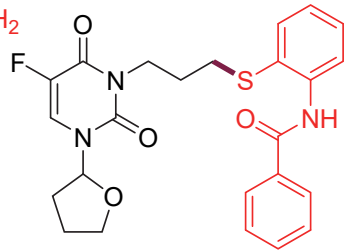

5j, $90 \%$<smiles>NN=CC(=NNN)SCCCn1c(=O)c(F)cn(C2CCCO2)c1=O</smiles>

5k, $91 \%$<smiles>O=c1c(F)cn(C2CCCO2)c(=O)n1CCCSCc1ccco1</smiles>

5I, $93 \%$<smiles>O=c1c(F)cn(C2CCCO2)c(=O)n1CCCSc1cccs1</smiles>

$5 \mathrm{~m}, 90 \%$<smiles>O=c1c(F)cn(C2CCCO2)c(=O)n1CCCSc1ccccn1</smiles>

5n, $83 \%$<smiles>O=c1c(F)cn(C2CCCO2)c(=O)n1CCSc1cn[nH]n1</smiles>

5s, $91 \%$<smiles>O=c1c(F)cn(C2CCCO2)c(=O)n1CCC[Se]c1ccccc1</smiles>

5t, $90 \%$<smiles>O=c1c(F)cn(C2CCCO2)c(=O)n1CCSc1ccccc1</smiles>

$n=1$ 5o, $94 \%$

$n=3$ 5p, $91 \%$ $n=4 \quad 5 q, 92 \%$<smiles>N#Cc1ccc(CCCn2c(=O)c(F)cn(C3CCCO3)c2=O)cc1</smiles>

$5 w, 95 \%$<smiles>O=c1c(F)cn(C2CCCO2)c(=O)n1CCC[Se]c1ccc(Cl)cc1</smiles>

5x, 93\%<smiles>COc1ccc([Se]CCCn2c(=O)c(F)cn(C3CCCO3)c2=O)cc1</smiles>

5u, $86 \%$

${ }^{a}$ Tegafluoroalkyl sulfonate (3): $1 \mathrm{mmol}$, diaryl disulfides or diselenides (4): $0.5 \mathrm{mmol}$, Ti catalyst: $5 \mathrm{~mol} \%, \mathrm{Zn}$ dust: $1.2 \mathrm{mmol}$, THF: $3 \mathrm{~mL}$, r.t., $\mathrm{N}_{2} ;{ }^{b}$ isolated yield.

显. 还考察了含不同杂环的芳基硫替加氟衍生物对两种 癌细胞的抑制活性. 结果显示, 含三唑基替加氟衍生物
的抑制作用比含 2-糠基、2-噻吩基及 2-吡啶基的替加氟 衍生物的抑制作用高 $(\mathbf{5 k} \sim \mathbf{5 n})$, 而且它们比大多数苯硫 
基替加氟衍生抑制活性高. 可能原因是杂环化合物自身 具有一定的生理活性，与替加氟的协同发挥作用，从而 增加了目标化合物对癌细胞的抑制活性. 对于化合物 5a, 5o 5r 而言, 随着替加氟烷基链长度的增加, 对 HCT116 细胞抑制作用也逐渐增加. 基于上述结果, 设 计合成了烷基侧链为正已基的三唑基硫替加氟衍生物 5s. 然而, 相比于化合物 5k 而言, 延长碳链后的正己基 三唑基硫替加氟衍生物对两种癌细胞的抑制作用增幅 不大. 最后, 考察了有机硒替加氟衍生物对上述两种癌 细胞的抑制作用. 结果表明, 有机硒替加氟衍生物对癌 细胞的抑制活性整体比有机硫替加氟衍生物活性强, 说 明引入硒可能比硫更有利于活性提高. 苯环含不同取代 基 $\left(\mathrm{CH}_{3}, \mathrm{OCH}_{3}, \mathrm{Cl}, \mathrm{CN}\right)$ 的有机硒替加氟衍生物均显示了 较高的抑制活性 $(\mathbf{5 u} \sim \mathbf{5 x})$. 此外, 苄基硒硒替加氟衍生 物比苯基硒替加氟衍生物具有较好的抑制活性. 对上述 含硫或硒的替加氟衍生物的药理实验及体内活性测试 还在进一步研究中.

表 3 替加氟衍生物抗肿瘤活性 ${ }^{a}$

Table 3 Antitumor activities of tegafur derivatives

\begin{tabular}{cll}
\hline \multirow{2}{*}{ Compd. } & \multicolumn{2}{c}{$\mathrm{IC}_{50}+\mathrm{SD} /\left(\mu \mathrm{mol} \cdot \mathrm{L}^{-1}\right)$} \\
\cline { 2 - 3 } Tegafur & $861.13 \pm 15.34$ & $1000.42 \pm 10.89$ \\
$\mathbf{5 a}$ & $518.74 \pm 4.38$ & $610.82 \pm 6.86$ \\
$\mathbf{5 b}$ & $223.15 \pm 2.09$ & $445.23 \pm 4.70$ \\
$\mathbf{5 c}$ & $389.53 \pm 3.79$ & $398.80 \pm 4.52$ \\
$\mathbf{5 d}$ & $217.06 \pm 1.74$ & $242.16 \pm 2.10$ \\
$\mathbf{5 e}$ & $994.99 \pm 4.57$ & $883.61 \pm 1.69$ \\
$\mathbf{5 f}$ & $176.72 \pm 3.16$ & $265.08 \pm 2.68$ \\
$\mathbf{5 g}$ & $447.89 \pm 3.94$ & $458.44 \pm 3.15$ \\
$\mathbf{5 h}$ & $1058.44 \pm 6.91$ & $501.28 \pm 7.84$ \\
$\mathbf{5 i}$ & $246.49 \pm 12.71$ & $238.43 \pm 1.53$ \\
$\mathbf{5 j}$ & $729.13 \pm 3.75$ & $348.14 \pm 6.50$ \\
$\mathbf{5 k}$ & $142.07 \pm 7.77$ & $240.11 \pm 4.66$ \\
$\mathbf{5 l}$ & $224.91 \pm 4.88$ & $274.50 \pm 3.32$ \\
$\mathbf{5 m}$ & $252.36 \pm 3.88$ & $290.93 \pm 6.12$ \\
$\mathbf{5 n}$ & $308.94 \pm 5.90$ & $258.75 \pm 2.19$ \\
$\mathbf{5 0}$ & $601.26 \pm 5.80$ & $1115.76 \pm 4.05$ \\
$\mathbf{5 p}$ & $428.59 \pm 3.78$ & $744.04 \pm 2.73$ \\
$\mathbf{5 q}$ & $352.59 \pm 2.62$ & $771.33 \pm 5.50$ \\
$\mathbf{5 r}$ & $290.06 \pm 3.49$ & $604.25 \pm 4.03$ \\
$\mathbf{5 s}$ & $135.34 \pm 1.88$ & $226.97 \pm 3.86$ \\
$\mathbf{5 t}$ & $392.60 \pm 2.03$ & $405.85 \pm 3.91$ \\
$\mathbf{5 u}$ & $239.64 \pm 2.40$ & $235.93 \pm 4.34$ \\
$\mathbf{5 v}$ & $223.40 \pm 2.97$ & $197.35 \pm 2.16$ \\
$\mathbf{5 w}$ & $224.85 \pm 4.32$ & $230.69 \pm 3.62$ \\
$\mathbf{5 x}$ & $200.62 \pm 2.92$ & $247.33 \pm 3.43$ \\
$\mathbf{5 y}$ & $198.56 \pm 3.30$ & $335.06 \pm 5.65$ \\
\hline
\end{tabular}

${ }^{a}$ Standard deviation, all compounds were dissolved in DMSO (dimethyl sulfoxide), all experiments were performed independently at least 3 times.
通过染色实验和流式细胞术探究替加氟衍生物 $\mathbf{5 k}$ 对 HCT116 细胞的抑制作用是否与细胞调亡有关. 如图 3 所示, 对照组只通过苂光染料 DAPI 对细胞进行染色; 而实验组用化合物 5k 处理 HCT116 细胞 $(100 \mu \mathrm{g} / \mathrm{mL})$, 经 $24 \mathrm{~h}$ 后，再用 DAPI 染色处理. 实验组细胞染色质发 生浓缩，细胞核碎裂成大小不等的圆形小体. 而染色质 浓缩和核碎裂是细胞调亡的一个特征，说明替加氟衍生 物 5k 可诱导细胞凋亡从而抑制细胞生长. 在上述研究 基础上, 采用 Annexin V-FITC/PI 染色结合流式细胞仪 定量检测浓度分别为 0,5 和 $30 \mu \mathrm{g} / \mathrm{mL}$ 的化合物 $\mathbf{5 k}$ 诱导 HCT116 细胞的调亡情况. 如图 4 所示, 在未经化合物 $\mathbf{5 k}$ 处理的情况下, 孵育 $24 \mathrm{~h}$ 后调亡细胞数量仅占总细 胞数的 $0.11 \%$. 若在低浓度 $5 \mu \mathrm{g} / \mathrm{mL}$ 的化合物 $\mathbf{5 k}$ 作用 HCT116 细胞后, HCT116 细胞的总调亡率增加到 1.64\%. 当化合物 5k 的浓度增加到 $30 \mu \mathrm{g} / \mathrm{mL}$ 时，早期和晚期凋 亡的总比值均相应升高，高达 $30.3 \%$. 可见，化合物 $\mathbf{5 k}$ 可浓度依赖性地诱导 HCT116 细胞调亡.

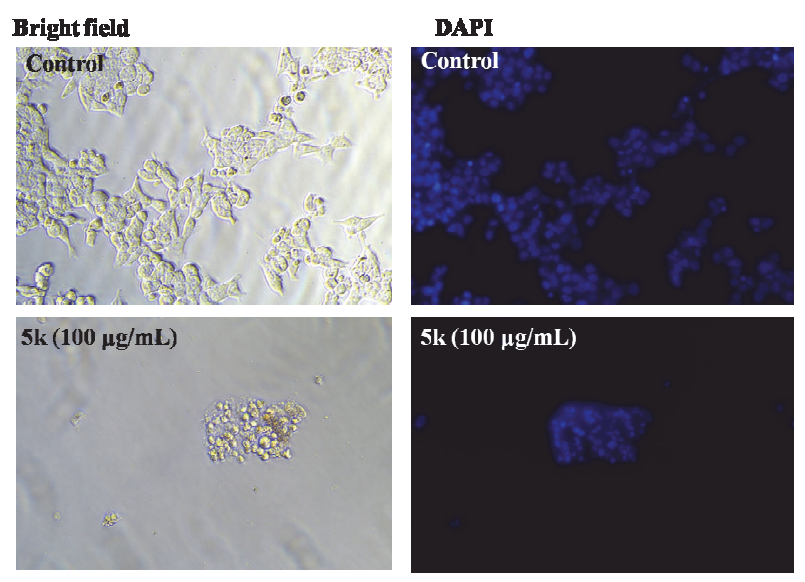

图 3 化合物 $\mathbf{5 k}$ 处理 HCT116 细胞的 DAPI 染色实验

Figure 3 DAPI staining experiment on HCT116 cells treated with compound $\mathbf{5 k}$

最后，评价了替加氟及其衍生物对正常人胚胎肾细 胞 293 (HEK 293)的毒性, 结果见图 5. 如图 5a 所示, 选 择化合物 5k 和替加氟分别在不同浓度下作用 HEK 293 细胞, 可发现在低浓度下 $(\leqslant 100 \mu \mathrm{g} / \mathrm{mL})$, 这两种化合物 对 HEK 293 细胞的抑制作用差别不大. 随着浓度逐渐增 加 $(\geqslant 150 \mu \mathrm{g} / \mathrm{mL})$, 两者抑制作用差别明显增大. 当浓度 为 $200 \mu \mathrm{g} / \mathrm{mL}$ 时，替加氟对 HEK 293 细胞抑制率超过 90\%，化合物 $\mathbf{5 k}$ 的抑制率仅为 $28 \%$. 因此，初步选择浓 度为 $200 \mu \mathrm{g} / \mathrm{mL}$ 考察有机硫(硒)替加氟衍生物对 HEK 293 细胞的毒性. 如图 5b 所示, 所有的化合物均比替加 氟的对 HEK 293 细胞的抑制作用小, 说明有机硫(硒)替 加氟衍生物比替加氟作用于 HEK 293 细胞的毒性低. 尤 其是含有三唑基的化合物 $5 \mathrm{k}$ 和 $5 \mathrm{~s}$ 显示了较低的毒性. 上述化合物的毒性分析还在进一步研究中. 

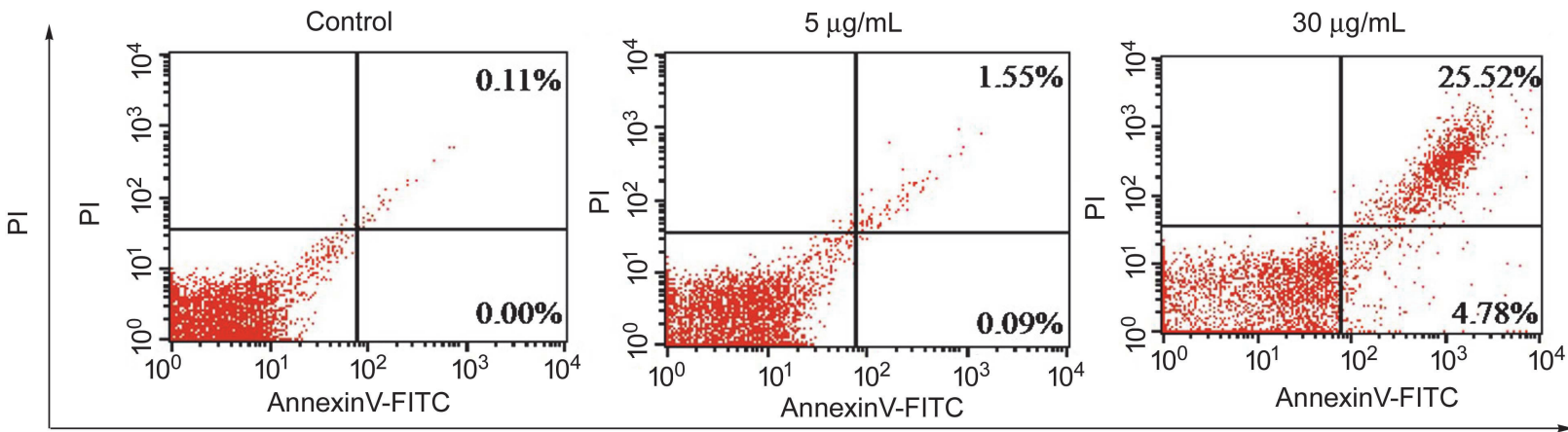

AnnexinV-FITC

图 4 流式细胞术检测化合物 $\mathbf{5 k}$ 诱导 HCT116 细胞调亡的影响

Figure 4 Effect of compound 5k on HCT116 cell apoptosis by flow cytometry
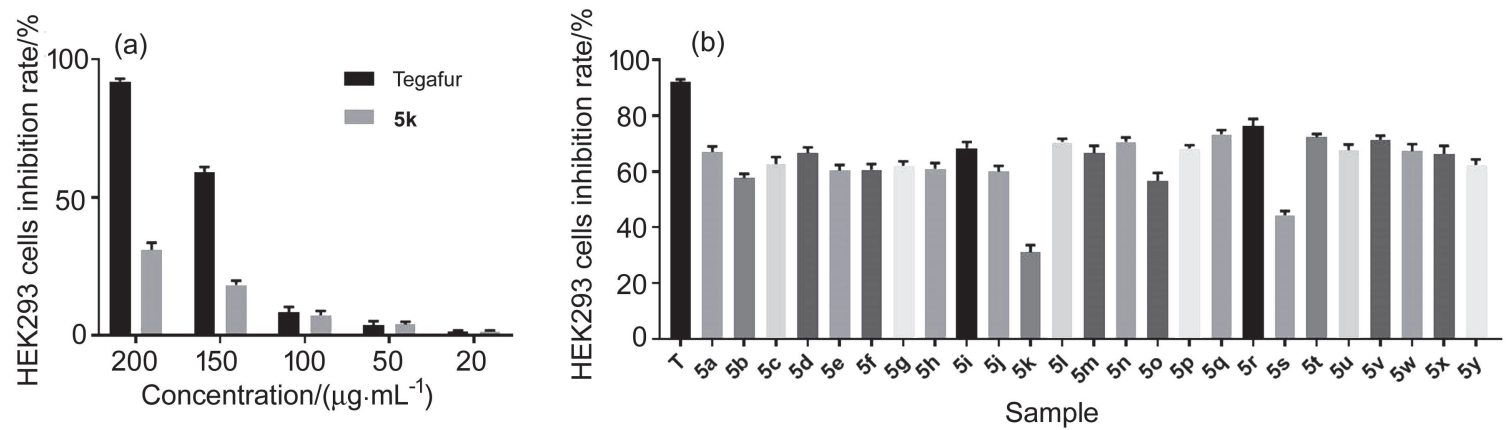

图 5 (a)在不同浓度下化合物 5k 和替加氟作用于 HEK293 细胞的抑制率, 及(b)有机硫(硒)替加氟衍生物对 HEK 293 细胞的抑制 率 $(200 \mu \mathrm{g} / \mathrm{mL})$

Figure 5 (a) Inhibition rate of compounds 5k and tegafur on HEK293 cells at different concentrations, and (b) inhibition rate of organic sulfur (selenium) tegafur derivative on HEK 293 cells $(200 \mu \mathrm{g} / \mathrm{mL})$

\section{2 结论}

综上所述，在双核钣全氟丁基磺酸配合物/锌粉催 化下，通过替加氟烷基磺酸酯与二芳基二硫(硒)醚反应， 成功合成了 25 种新型芳基 $\left(N^{3}\right.$-替加氟烷基)硫(硒)醚衍 生物. 由于烷基和含硫或硒基团的引入, 绝大多数目标 化合物对结肠癌细胞 HCT116 和胃癌细胞 SGC-7901 的 抑制作用高于替加氟. 细胞染色实验和流式细胞仪定量 检测实验证实了替加氟衍生物可诱导细胞调亡, 从而抑 制细胞生长. 有机硫(硒)替加氟衍生物作用与正常人胚 胎肾细胞 HEK 293 的毒性比替加氟低(浓度为 200 $\mu \mathrm{g} / \mathrm{mL}$ ). 总之, 本文提供了一种新型有机硫(硒)替加氟 衍生物的的合成方法, 并进行了体外抗肿瘤活性研究, 为后续替加氟药物修饰及药物活性研究提供重要参考.

\section{3 实验部分}

\section{1 仪器与试剂}

${ }^{1} \mathrm{H}$ NMR 和 ${ }^{13} \mathrm{C}$ NMR 用 BRUKER AVANCE II 400 型仪测定 $\left[\mathrm{CDCl}_{3}\right.$ 为溶剂, 三甲硅烷基(TMS)作为内标]; 质谱由 Thermo Scientific Q Exactive 型仪测定. 低速离 心机(湖南湘仪实验室仪器开发有限公司), 荧光倒置显
微镜(Nikon), 二氧化碳培养箱(eppendorf), 超净工作台 (上海智城分析仪器制造有限公司). 替加氟、2-氯乙醇、 3-氯-1-丙醇、4-氯-1-丁醇、5-氯-1-戊醇、6-氯-1-己醇、 对甲苯磺酰氯、三乙胺、二芳基二硫醚、二芳基二硒梄、 四氢呋喃、乙腈、三乙胺和锌粉等均从艾览化工科技有 限公司和百灵威试剂公司购买. 有机钛全氟丁基磺酸盐 按照文献合成 ${ }^{[26]}$. 硅胶为青岛海洋化工厂产品. 两个细 胞株结肠癌细胞 HCT116、胃癌细胞 SGC-7901 和正常 人胚胎肾细胞 HEK 293 均由中国科学院上海有机化学研 究所细胞库提供. DMEM (Dulbecco's Modified Eagle Media)高糖培养基和磷酸盐缓冲液(PBS)从新赛美生物 科技有限公司购买.

\section{2 实验方法}

\subsubsection{N-(3-羟烷基) 替加氟的合成}

向 $50 \mathrm{~mL}$ 圆底烧瓶中依次加入替加氟 $(2.5 \mathrm{mmol}, 0.5$ $\mathrm{g}) 、$ 氯代烷基醇 $(3 \mathrm{mmol}) 、 \mathrm{NaHCO}_{3}(0.4 \mathrm{~g})$ 和乙腈 $10 \mathrm{~mL}$, 加热回流反应 $12 \mathrm{~h}$. 反应结束后[薄层色谱(TLC)追踪反 应], 过滤, 滤液减压旋干, 柱层析分离[洗脱剂: 二氯甲 烷/乙酸乙酯, $V: V=1: 1]$ 得到化合物 $\mathbf{2}^{[27]}$.

$N^{3}$-(2-羟基乙基)替加氟(2a) $)^{[9]}$ : 产率 $92 \%$, 白色固 体, m.p. 93 94 ${ }^{\circ} \mathrm{C} ;{ }^{1} \mathrm{H}$ NMR $\left(400 \mathrm{MHz}, \mathrm{CDCl}_{3}\right) \delta: 7.43$ 
$(\mathrm{d}, J=5.6 \mathrm{~Hz}, 1 \mathrm{H}), 6.00 \sim 5.98(\mathrm{~m}, 1 \mathrm{H}), 4.22(\mathrm{t}, J=5.4$ $\mathrm{Hz}, 2 \mathrm{H}), 4.00$ (q, $J=5.2 \mathrm{~Hz}, 1 \mathrm{H}), 3.88$ (q, $J=5.6 \mathrm{~Hz}, 2 \mathrm{H})$, $2.47 \sim 2.38(\mathrm{~m}, 2 \mathrm{H}), 2.09 \sim 2.03(\mathrm{~m}, 2 \mathrm{H}), 1.97 \sim 1.89(\mathrm{~m}$, 1H), $1.75(\mathrm{~s}, 1 \mathrm{H}) ;{ }^{13} \mathrm{C}$ NMR (100 MHz, $\left.\mathrm{CDCl}_{3}\right) \delta: 157.8$ (d, $J=25.3 \mathrm{~Hz}), 149.8,139.9$ (d, $J=232.4 \mathrm{~Hz}), 122.0$ (d, $J=33.8 \mathrm{~Hz}), 88.3,70.4,61.1,44.0,33.0,23.7$; TOFHRMS (ESI) calcd for $\mathrm{C}_{10} \mathrm{H}_{13} \mathrm{FN}_{2} \mathrm{O}_{4}[\mathrm{M}+\mathrm{H}]^{+}$244.0859, found 244.0851 .

$N^{3}$-(3-差基正丙基)替加氟(2b) ${ }^{[9]}$ : 产率 $88 \%$, 白色固 体, m.p. $67 \sim 68{ }^{\circ} \mathrm{C} ;{ }^{1} \mathrm{H}$ NMR $\left(400 \mathrm{MHz}, \mathrm{CDCl}_{3}\right) \delta: 7.43$ $(\mathrm{d}, J=5.6 \mathrm{~Hz}, 1 \mathrm{H}), 5.94$ (d, $J=4.8 \mathrm{~Hz}, 1 \mathrm{H}), 4.27 \sim 4.18$ $(\mathrm{m}, 1 \mathrm{H}), 4.07 \sim 4.03(\mathrm{~m}, 2 \mathrm{H}), 4.00 \sim 3.93(\mathrm{~m}, 1 \mathrm{H}), 3.54 \sim$ $3.48(\mathrm{~m}, 2 \mathrm{H}), 3.11(\mathrm{~s}, 1 \mathrm{H}), 2.42 \sim 2.34(\mathrm{~m}, 1 \mathrm{H}), 2.07 \sim$ $1.98(\mathrm{~m}, 2 \mathrm{H}), 1.95 \sim 1.80(\mathrm{~m}, 3 \mathrm{H}) ;{ }^{13} \mathrm{C} \mathrm{NMR}(100 \mathrm{MHz}$, $\left.\mathrm{CDCl}_{3}\right) \delta: 156.3(\mathrm{~d}, J=25.0 \mathrm{~Hz}), 149.3,139.8(\mathrm{~d}, \quad J=$ $232.5 \mathrm{~Hz}$ ), 122.0 (d, $J=33.8 \mathrm{~Hz}$ ), 88.2, 70.3, 58.8, 38.4, 32.9, 30.4, 23.7; TOF-HRMS (ESI) calcd for $\mathrm{C}_{11} \mathrm{H}_{16} \mathrm{FN}_{2} \mathrm{O}_{4}$ $[\mathrm{M}+\mathrm{H}]^{+}$259.1094, found 259.1090.

$N^{3}$-(3-着基正丁基)替加氟(2c) ${ }^{[9]}$ : 产率 $80 \%$, 白色固 体, m.p. $65 \sim 66{ }^{\circ} \mathrm{C} ;{ }^{1} \mathrm{H}$ NMR $\left(400 \mathrm{MHz}, \mathrm{CDCl}_{3}\right) \delta: 7.40$ $(\mathrm{d}, J=6.4 \mathrm{~Hz}, 1 \mathrm{H}), 6.02 \sim 6.00(\mathrm{~m}, 1 \mathrm{H}), 4.27 \sim 4.22(\mathrm{~m}$, $1 \mathrm{H}), 4.05 \sim 3.98(\mathrm{~m}, 3 \mathrm{H}), 3.72(\mathrm{~d}, J=6.6 \mathrm{~Hz}, 2 \mathrm{H}), 2.48 \sim$ $2.40(\mathrm{~m}, 1 \mathrm{H}), 2.13 \sim 2.04(\mathrm{~m}, 2 \mathrm{H}), 1.98 \sim 1.90(\mathrm{~m}, 1 \mathrm{H})$, $1.82 \sim 1.72(\mathrm{~m}, 2 \mathrm{H}), 1.68 \sim 1.59(\mathrm{~m}, 2 \mathrm{H}) ;{ }^{13} \mathrm{C}$ NMR $(100$ $\left.\mathrm{MHz}, \mathrm{CDCl}_{3}\right) \delta: 157.4(\mathrm{~d}, J=25.9 \mathrm{~Hz}), 149.0,140.4$ (d, $J=234.6 \mathrm{~Hz}), 123.5$ (d, $J=33.7 \mathrm{~Hz}), 87.4,70.2$, 62.5, 45.1, 32.5, 29.2, 25.6, 23.8; TOF-HRMS (ESI) calcd for $\mathrm{C}_{12} \mathrm{H}_{18} \mathrm{FN}_{2} \mathrm{O}_{4}[\mathrm{M}+\mathrm{H}]^{+}$273.1251, found 273.1247.

$N^{3}$-(5-羟基正戍基)替加氟(2d): 产率 $85 \%$, 白色固 体, m.p. $107 \sim 108{ }^{\circ} \mathrm{C} ;{ }^{1} \mathrm{H}$ NMR (400 MHz, $\left.\mathrm{CDCl}_{3}\right) \delta$ : $7.39(\mathrm{~d}, J=5.6 \mathrm{~Hz}, 1 \mathrm{H}), 6.00(\mathrm{~d}, J=4.8 \mathrm{~Hz}, 1 \mathrm{H}), 4.26 \sim$ $4.20(\mathrm{~m}, 1 \mathrm{H}), 3.98 \sim 3.92(\mathrm{~m}, 2 \mathrm{H}), 3.65(\mathrm{q}, J=6.0 \mathrm{~Hz}$, $2 \mathrm{H}), 2.47 \sim 2.38(\mathrm{~m}, 1 \mathrm{H}), 2.09 \sim 2.03(\mathrm{~m}, 2 \mathrm{H}), 1.96 \sim 1.90$ $(\mathrm{m}, 1 \mathrm{H}), 1.72 \sim 1.59(\mathrm{~m}, 6 \mathrm{H}), 1.45 \sim 1.39(\mathrm{~m}, 2 \mathrm{H}) ;{ }^{13} \mathrm{C}$ NMR (100 MHz, $\left.\mathrm{CDCl}_{3}\right) \delta: 157.1(\mathrm{~d}, J=25.1 \mathrm{~Hz}), 149.1$, $139.8(\mathrm{~d}, J=232.3 \mathrm{~Hz}), 121.6(\mathrm{~d}, J=33.8 \mathrm{~Hz}), 88.0,70.3$, 62.6, 41.6, 33.0, 32.2, 27.2, 23.8, 23.0; TOF-HRMS (ESI) calcd for $\mathrm{C}_{13} \mathrm{H}_{19} \mathrm{FN}_{2} \mathrm{O}_{4}[\mathrm{M}+\mathrm{H}]^{+}$286.1329, found 286.1323 .

$N^{3}$-(6-着基正己基)替加氟(2e ${ }^{[24]}$ : 产率 $84 \%$, 白色 固体, m.p. $77 \sim 78{ }^{\circ} \mathrm{C} ;{ }^{1} \mathrm{H}$ NMR (400 MHz, $\left.\mathrm{CDCl}_{3}\right) \delta$ : $7.39(\mathrm{~d}, J=5.6 \mathrm{~Hz}, 1 \mathrm{H}), 6.00(\mathrm{~d}, J=4.8 \mathrm{~Hz}, 1 \mathrm{H}), 4.26 \sim$ $4.20(\mathrm{~m}, 1 \mathrm{H}), 4.03 \sim 3.91(\mathrm{~m}, 3 \mathrm{H}), 3.63(\mathrm{q}, J=5.6 \mathrm{~Hz}$, $2 \mathrm{H}), 2.45 \sim 2.38(\mathrm{~m}, 1 \mathrm{H}), 2.10 \sim 2.02(\mathrm{~m}, 2 \mathrm{H}), 1.95 \sim 1.89$ $(\mathrm{m}, 1 \mathrm{H}), 1.65 \sim 1.55(\mathrm{~m}, 5 \mathrm{H}), 1.42 \sim 1.37(\mathrm{~m}, 4 \mathrm{H}) ;{ }^{13} \mathrm{C}$
NMR (100 MHz, $\left.\mathrm{CDCl}_{3}\right) \delta: 157.2(\mathrm{~d}, J=25.1 \mathrm{~Hz}), 149.1$, 139.9 (d, $J=232.5 \mathrm{~Hz}), 121.5$ (d, $J=33.8 \mathrm{~Hz}), 88.0,70.3$, 62.7, 41.6, 33.0, 32.5, 27.4, 26.4, 25.2, 23.8; TOF-HRMS (ESI) calcd for $\mathrm{C}_{14} \mathrm{H}_{21} \mathrm{FN}_{2} \mathrm{O}_{4}[\mathrm{M}+\mathrm{H}]^{+} 300.1458$, found 300.1453 .

\section{2 .2 替加氟烷基磺酸酯的合成}

向 $100 \mathrm{~mL}$ 的三颈烧瓶中依次加入对甲苯磺酰氯 (2.3 mmol, $439 \mathrm{mg}) 、$ DMAP (15.6 mg)、三乙胺 $(0.5 \mathrm{~mL})$ 和 $10 \mathrm{~mL}$ 二氯甲烷. 然后置于冰浴下搅拌 $0.5 \mathrm{~h}$. 在恒压 漏斗中加入 $N$-(羟烷基)替加氟(2 mmol)和二氯甲烷(10 $\mathrm{mL}$ ), 并将恒压漏斗置于三颈烧瓶上, 在冰水浴下缓慢 滴加, 约 $0.5 \mathrm{~h}$ 滴加完毕. 逐渐升至室温反应 $10 \mathrm{~h}$ (TLC 跟踪). 反应完毕后, 加入 $0.2 \mathrm{~mol} / \mathrm{L} \mathrm{HCl}(10 \mathrm{~mL})$, 分液. 水层用二氯甲烷萃取 $(15 \mathrm{~mL} \times 3)$, 然后合并有机相. 用 无水硫酸钠干燥, 过滤, 滤液减压旋干, 柱层析分离(洗 脱剂: 乙酸乙酯/石油醚, $V: V=1: 1$ )得化合物 $\mathbf{3}^{[25]}$.

对甲苯磺酸 $\left(N^{3}\right.$-替加氟)乙酯 $(3 \mathbf{a})^{[25]}$ : 产率 $92 \%$, 白 色固体, m.p. 95 96 ${ }^{\circ} \mathrm{C} ;{ }^{1} \mathrm{H}$ NMR (400 MHz, $\left.\mathrm{CDCl}_{3}\right) \delta$ : $8.01(\mathrm{~s}, 1 \mathrm{H}), 7.66(\mathrm{~d}, J=7.2 \mathrm{~Hz}, 1 \mathrm{H}), 7.81 \sim 7.78(\mathrm{~m}, 1 \mathrm{H})$, $7.43 \sim 7.34(\mathrm{~m}, 2 \mathrm{H}), 5.99 \sim 5.98(\mathrm{~m}, 1 \mathrm{H}), 4.47 \sim 4.35(\mathrm{~m}$, $2 \mathrm{H}), 4.32 \sim 4.26(\mathrm{~m}, 1 \mathrm{H}), 4.24 \sim 4.22(\mathrm{~m}, 2 \mathrm{H}), 2.45 \sim 2.40$ $(\mathrm{m}, 3 \mathrm{H}), 2.11 \sim 1.89(\mathrm{~m}, 3 \mathrm{H}) ;{ }^{13} \mathrm{C} \mathrm{NMR}\left(100 \mathrm{MHz}, \mathrm{CDCl}_{3}\right)$ $\delta$ : $157.0(\mathrm{~d}, J=25.3 \mathrm{~Hz}), 148.9,139.8(\mathrm{~d}, J=232.3 \mathrm{~Hz})$, 136.3, 128.2, 127.3, 125.5, 122.0 (d, $J=33.7 \mathrm{~Hz}), 87.7$, 69.9, 67.6, 42.6, 32.3, 23.3, 20.8; TOF-HRMS (ESI) calcd for $\mathrm{C}_{17} \mathrm{H}_{19} \mathrm{FN}_{2} \mathrm{O}_{6} \mathrm{~S}[\mathrm{M}+\mathrm{H}]^{+}$398.0948, found 398.0954.

对甲苯磺酸 $\left(N^{3}\right.$-替加氟)正丙酯 $(\mathbf{3 b})$ : 产率 $91 \%$, 白 色固体, m.p. 77 78 ${ }^{\circ} \mathrm{C} ;{ }^{1} \mathrm{H}$ NMR (400 MHz, $\left.\mathrm{CDCl}_{3}\right) \delta$ : 7.75 (d, $J=8.4 \mathrm{~Hz}, 2 \mathrm{H}), 7.41$ (d, $J=6.0 \mathrm{~Hz}, 1 \mathrm{H}), 7.34$ (d, $J=8.0 \mathrm{~Hz}, 2 \mathrm{H}), 5.98 \sim 5.96(\mathrm{~m}, 1 \mathrm{H}), 4.26 \sim 4.20(\mathrm{~m}, 1 \mathrm{H})$, $4.09(\mathrm{t}, J=6.2 \mathrm{~Hz}, 2 \mathrm{H}), 4.02 \sim 3.96(\mathrm{~m}, 2 \mathrm{H}), 2.45(\mathrm{~s}, 3 \mathrm{H})$, $2.41 \sim 2.35(\mathrm{~m}, 1 \mathrm{H}), 2.12 \sim 1.88(\mathrm{~m}, 5 \mathrm{H}) ;{ }^{13} \mathrm{C}$ NMR $(100$ $\left.\mathrm{MHz}, \mathrm{CDCl}_{3}\right): \delta 157.0(\mathrm{~d}, J=25.3 \mathrm{~Hz}), 148.9,144.8$, $139.8(\mathrm{~d}, J=232.3 \mathrm{~Hz}), 132.8,129.8,127.9,122.0$ (d, $J=$ $33.7 \mathrm{~Hz}$ ), 88.1, 70.3, 68.4, 38.5, 32.9, 27.0, 23.7, 21.6; TOF-HRMS (ESI) calcd for $\mathrm{C}_{18} \mathrm{H}_{22} \mathrm{FN}_{2} \mathrm{O}_{6} \mathrm{~S}[\mathrm{M}+\mathrm{H}]^{+}$, 413.1183, found 413.1178.

对甲苯磺酸 $\left(N^{3}\right.$-替加氟)正丁酯(3c): 产率 $88 \%$, 白 色固体, m.p. $72 \sim 73{ }^{\circ} \mathrm{C} ;{ }^{1} \mathrm{H}$ NMR (400 MHz, $\left.\mathrm{CDCl}_{3}\right) \delta$ : 7.80 (d, $J=8.4 \mathrm{~Hz}, 2 \mathrm{H}), 7.40$ (d, $J=6.0 \mathrm{~Hz}, 1 \mathrm{H}), 7.36$ (d, $J=8.0 \mathrm{~Hz}, 2 \mathrm{H}), 6.00 \sim 5.98(\mathrm{~m}, 1 \mathrm{H}), 4.27 \sim 4.22(\mathrm{~m}, 1 \mathrm{H})$, $4.08 \sim 3.99(\mathrm{~m}, 3 \mathrm{H}), 3.93 \sim 3.90(\mathrm{~m}, 2 \mathrm{H}), 2.47(\mathrm{~s}, 3 \mathrm{H})$, $2.45 \sim 2.41(\mathrm{~m}, 1 \mathrm{H}), 2.12 \sim 2.04(\mathrm{~m}, 2 \mathrm{H}), 1.98 \sim 1.92(\mathrm{~m}$, 1H), $1.74 \sim 1.69(\mathrm{~m}, 1 \mathrm{H}) ;{ }^{13} \mathrm{C}$ NMR $\left(100 \mathrm{MHz}, \mathrm{CDCl}_{3}\right) \delta$ : $157.1(\mathrm{~d}, J=25.4 \mathrm{~Hz}), 148.9,144.9,139.8$ (d, $J=232.3$ 
$\mathrm{Hz}), 132.7,127.9,122.1(\mathrm{~d}, J=33.8 \mathrm{~Hz}), 88.2,70.4$, 68.5, 38.6, 33.0, 29.7, 26.9, 23.7, 21.6; TOF-HRMS (ESI) calcd for $\mathrm{C}_{19} \mathrm{H}_{24} \mathrm{~F}-\mathrm{N}_{2} \mathrm{O}_{6} \mathrm{~S}[\mathrm{M}+\mathrm{H}]^{+}$427.1339, found 427.1334 .

对甲苯磺酸 $\left(N^{3}\right.$-替加氟)正戊酯(3d): 产率 $90 \%$, 白 色固体, m.p. 69 70 ${ }^{\circ} \mathrm{C} ;{ }^{1} \mathrm{H}$ NMR $\left(400 \mathrm{MHz}, \mathrm{CDCl}_{3}\right) \delta$ : $7.78(\mathrm{~d}, J=8.4 \mathrm{~Hz}, 2 \mathrm{H}), 7.42 \sim 7.41(\mathrm{~m}, 1 \mathrm{H}), 7.36(\mathrm{~d}, J=$ $8.0 \mathrm{~Hz}, 2 \mathrm{H}), 5.98$ (t, $J=2.4 \mathrm{~Hz}, 1 \mathrm{H}), 4.26 \sim 4.21(\mathrm{~m}, 1 \mathrm{H})$, $4.01(\mathrm{t}, J=6.4 \mathrm{~Hz}, 2 \mathrm{H}), 3.91 \sim 3.86(\mathrm{~m}, 2 \mathrm{H}), 2.46(\mathrm{~s}, 3 \mathrm{H})$, $2.43 \sim 2.37(\mathrm{~m}, 1 \mathrm{H}), 2.11 \sim 2.02(\mathrm{~m}, 3 \mathrm{H}), 1.99 \sim 1.90(\mathrm{~m}$, $1 \mathrm{H}), 1.74 \sim 1.64(\mathrm{~m}, 3 \mathrm{H}), 1.59 \sim 1.57(\mathrm{~m}, 2 \mathrm{H}), 1.40 \sim 1.33$ $(\mathrm{m}, 2 \mathrm{H}) ;{ }^{13} \mathrm{C}$ NMR $\left(100 \mathrm{MHz}, \mathrm{CDCl}_{3}\right) \delta: 157.1(\mathrm{~d}, J=25.1$ $\mathrm{Hz}), 148.9$, 144.8, 139.1 (d, $J=232.0 \mathrm{~Hz}), 133.0,127.8$, $121.8(\mathrm{~d}, J=33.8 \mathrm{~Hz}), 88.0,70.3,63.7,41.2,33.0,26.8$, 23.8, 22.7, 21.6; TOF-HRMS (ESI) calcd for $\mathrm{C}_{20} \mathrm{H}_{25} \mathrm{~F}-$ $\mathrm{N}_{2} \mathrm{O}_{6} \mathrm{~S}[\mathrm{M}+\mathrm{H}]^{+}$440.1417, found 440.1425.

对甲苯磺酸 $\left(N^{3}\right.$-替加氟)正己酯 $(3 \mathbf{e})$ ：产率 $91 \%$, 白 色固体, m.p. $67 \sim 68{ }^{\circ} \mathrm{C} ;{ }^{1} \mathrm{H}$ NMR $\left(400 \mathrm{MHz}, \mathrm{CDCl}_{3}\right) \delta$ : 7.78 (d, $J=8.4 \mathrm{~Hz}, 2 \mathrm{H}), 7.41(\mathrm{~d}, J=6.0 \mathrm{~Hz}, 1 \mathrm{H}), 7.36$ (d, $J=8.8 \mathrm{~Hz}, 2 \mathrm{H}), 6.00(\mathrm{t}, J=5.2 \mathrm{~Hz}, 1 \mathrm{H}), 4.27 \sim 4.21(\mathrm{~m}$, $1 \mathrm{H}), 4.00(\mathrm{t}, J=6.4 \mathrm{~Hz}, 2 \mathrm{H}), 3.91 \sim 3.86(\mathrm{~m}, 2 \mathrm{H}), 2.46(\mathrm{~s}$, $3 \mathrm{H}), 2.43 \sim 2.37(\mathrm{~m}, 1 \mathrm{H}), 2.11 \sim 2.02(\mathrm{~m}, 3 \mathrm{H}), 1.99 \sim 1.90$ $(\mathrm{m}, 1 \mathrm{H}), 1.69 \sim 1.55(\mathrm{~m}, 5 \mathrm{H}), 1.43 \sim 1.25(\mathrm{~m}, 6 \mathrm{H}) ;{ }^{13} \mathrm{C}$ NMR (100 MHz, $\left.\mathrm{CDCl}_{3}\right): \delta 157.1(\mathrm{~d}, J=25.1 \mathrm{~Hz}), 148.9$, 144.8, 139.9 (d, $J=232.0 \mathrm{~Hz}), 133.0,129.9,127.8,121.7$ (d, $J=33.8 \mathrm{~Hz}), 88.0,70.4,41.4,32.9,28.6,27.2,26.1$, 25.0, 23.8, 21.6; TOF-HRMS (ESI) calcd for $\mathrm{C}_{21} \mathrm{H}_{27} \mathrm{~F}-$ $\mathrm{N}_{2} \mathrm{O}_{6} \mathrm{~S}[\mathrm{M}+\mathrm{H}]^{+}$454.1574, found 454.1581.

\subsection{3 有机硫(硒)替加氟衍生物的合成}

氮气保护下，向 $25 \mathrm{~mL}$ 反应管中依次加入有机钛全 氟丁基磺酸配合物(68.7 mg, $5 \mathrm{~mol} \%$ )、活化的锌粉( 80 $\mathrm{mg}, 1.2 \mathrm{mmol}$ 、替加氟烷基磺酸酯 $(1 \mathrm{mmol})$ 和二芳基二 硫(硒)醚(0.5 mmol)和 THF (3 mL). 室温下搅拌 1 h. TLC 跟踪反应完成. 减压旋干溶剂, 柱层析分离(洗脱剂: 乙 酸乙酯/石油醚, $V: V=1: 5)$, 得目标化合物 5 .

苯基( $N^{3}$-替加氟)正丙基硫醚(5a): 产率 94\%, 无色 油状液体. ${ }^{1} \mathrm{H}$ NMR (400 MHz, $\left.\mathrm{CDCl}_{3}\right) \delta: 7.41(\mathrm{~d}, J=6.0$ $\mathrm{Hz}, 1 \mathrm{H}), 7.38$ (d, $J=8.0 \mathrm{~Hz}, 2 \mathrm{H}), 7.31$ (t, $J=7.6 \mathrm{~Hz}, 2 \mathrm{H})$, $7.21(\mathrm{~d}, \quad J=7.2 \mathrm{~Hz}, 1 \mathrm{H}), 6.01 \sim 6.00(\mathrm{~m}, 1 \mathrm{H}), 4.27 \sim 4.21$ (m, $1 \mathrm{H}), 4.13(\mathrm{t}, J=7.0 \mathrm{~Hz}, 2 \mathrm{H}), 4.05 \sim 3.99(\mathrm{~m}, 1 \mathrm{H}), 2.98$ $(\mathrm{t}, J=7.4 \mathrm{~Hz}, 2 \mathrm{H}), 2.46 \sim 2.39(\mathrm{~m}, 1 \mathrm{H}), 2.13 \sim 1.92(\mathrm{~m}$, $5 \mathrm{H}) ;{ }^{13} \mathrm{C}$ NMR $\left(100 \mathrm{MHz}, \mathrm{CDCl}_{3}\right) \delta: 157.0$ (d, $J=25.3$ $\mathrm{Hz}), 149.0,139.9$ (d, $J=232.5 \mathrm{~Hz}), 136.0,129.7,128.9$, 126.2, 122.0 (d, $J=33.8 \mathrm{~Hz}), 88.0,70.3,40.8,33.0,31.5$, 27.2, 23.8; TOF-HRMS (ESI) calcd for $\mathrm{C}_{17} \mathrm{H}_{20} \mathrm{FN}_{2} \mathrm{O}_{3} \mathrm{~S}$
$[\mathrm{M}+\mathrm{H}]^{+}$351.1179, found 351.1183.

邻氟苯基( $N^{3}$-替加氟)正丙基硫醚 $(\mathbf{5 b})$ ：产率 92\%, 淡黄色油状液体. ${ }^{1} \mathrm{H}$ NMR (400 $\left.\mathrm{MHz}, \mathrm{CDCl}_{3}\right) \delta$ : 7.42 $7.38(\mathrm{~m}, 2 \mathrm{H}), 7.24 \sim 7.19(\mathrm{~m}, 1 \mathrm{H}), 7.11 \sim 7.02(\mathrm{~m}, 2 \mathrm{H})$, $5.99 \sim 5.97(\mathrm{~m}, 1 \mathrm{H}), 4.23 \sim 4.21(\mathrm{~m}, 1 \mathrm{H}), 4.08(\mathrm{t}, J=6.8$ $\mathrm{Hz}, 2 \mathrm{H}), 4.03 \sim 3.97(\mathrm{~m}, 1 \mathrm{H}), 2.94(\mathrm{t}, J=7.4 \mathrm{~Hz}, 2 \mathrm{H})$, $2.45 \sim 2.37(\mathrm{~m}, 1 \mathrm{H}), 2.11 \sim 2.01(\mathrm{~m}, 2 \mathrm{H}), 1.99 \sim 1.88(\mathrm{~m}$, $3 \mathrm{H}) ;{ }^{13} \mathrm{C} \mathrm{NMR}\left(100 \mathrm{MHz}, \mathrm{CDCl}_{3}\right) \delta: 160.4,157.1(\mathrm{~d}, J=$ $25.3 \mathrm{~Hz}), 149.0,139.9$ (d, $J=232.4 \mathrm{~Hz}), 132.7,128.7$, $121.6(\mathrm{~d}, J=33.8 \mathrm{~Hz}), 115.7$ (d, $J=22.3 \mathrm{~Hz}), 88.1,70.4$, 40.7, 33.0, 31.1, 31.0, 27.2, 23.8; TOF-HRMS (ESI) calcd for $\mathrm{C}_{17} \mathrm{H}_{18} \mathrm{~F}_{2} \mathrm{~N}_{2} \mathrm{O}_{3} \mathrm{~S}[\mathrm{M}+\mathrm{H}]^{+}$368.1006, found 368.1010.

对氯苯基( $N^{3}$-替加氟)正丙基硫醚 $(5 \mathbf{c})$ ：产率 95\%, 白色固体; m.p. $112 \sim 114{ }^{\circ} \mathrm{C}$; ${ }^{1} \mathrm{H}$ NMR $(400 \mathrm{MHz}$, $\left.\mathrm{CDCl}_{3}\right) \delta: 7.42(\mathrm{~d}, J=4.6 \mathrm{~Hz}, 1 \mathrm{H}), 7.33 \sim 7.27(\mathrm{~m}, 4 \mathrm{H})$, $6.02 \sim 5.99(\mathrm{~m}, 1 \mathrm{H}), 4.28 \sim 4.23(\mathrm{~m}, 1 \mathrm{H}), 4.12 \sim 4.08(\mathrm{~m}$, $2 \mathrm{H}), 4.05 \sim 3.99(\mathrm{~m}, 1 \mathrm{H}), 2.95(\mathrm{t}, J=7.2 \mathrm{~Hz}, 2 \mathrm{H}), 2.47 \sim$ $2.40(\mathrm{~m}, 1 \mathrm{H}), 2.10 \sim 1.91(\mathrm{~m}, 5 \mathrm{H}) ;{ }^{13} \mathrm{C} \mathrm{NMR}(100 \mathrm{MHz}$, $\left.\mathrm{CDCl}_{3}\right) \delta: 157.1(\mathrm{~d}, J=25.3 \mathrm{~Hz}), 149.0,139.9$ (d, $J=232.7$ $\mathrm{Hz}), 134.4,132.3,131.2,129.0,128.9,126.2,122.1$ (d, $J=$ $33.7 \mathrm{~Hz}), 88.0,70.3,40.7$, 33.0. 31.8, 27.0, 23.8; TOFHRMS (ESI) calcd for $\mathrm{C}_{17} \mathrm{H}_{19} \mathrm{ClFN}_{2} \mathrm{O}_{3} \mathrm{~S} \quad[\mathrm{M}+\mathrm{H}]^{+}$ 385.0789, found 385.0785.

对硝基苯基( $N^{3}$-替加氟)正丙基硫醚(5d)：产率 94\%， 黄色油状液体. ${ }^{1} \mathrm{H}$ NMR $\left(400 \mathrm{MHz}, \mathrm{CDCl}_{3}\right) \delta: 8.15$ (d, $J=8.8 \mathrm{~Hz}, 2 \mathrm{H}), 7.44(\mathrm{~d}, J=6.0 \mathrm{~Hz}, 1 \mathrm{H}), 7.36$ (d, $J=6.8$ $\mathrm{Hz}, 2 \mathrm{H}), 6.03 \sim 6.01(\mathrm{~m}, 1 \mathrm{H}), 4.29 \sim 4.24(\mathrm{~m}, 1 \mathrm{H}), 4.18 \sim$ $4.11(\mathrm{~m}, 2 \mathrm{H}), 4.08 \sim 4.00(\mathrm{~m}, 1 \mathrm{H}), 3.11(\mathrm{t}, J=7.4 \mathrm{~Hz}, 2 \mathrm{H})$, $2.50 \sim 2.41(\mathrm{~m}, 1 \mathrm{H}), 2.13 \sim 1.92(\mathrm{~m}, 5 \mathrm{H}) ;{ }^{13} \mathrm{C}$ NMR $(100$ $\left.\mathrm{MHz}, \mathrm{CDCl}_{3}\right) \delta: 157.1(\mathrm{~d}, J=24.3 \mathrm{~Hz}), 149.0,147.0$, $145.2,139.9$ (d, $J=233.0 \mathrm{~Hz}), 126.4,124.0,122.1$ (d, $J=$ $33.7 \mathrm{~Hz}), 88.1,70.3,40.6,33.0,29.7,26.7$, 23.8; TOFHRMS (ESI) calcd for $\mathrm{C}_{17} \mathrm{H}_{19} \mathrm{FN}_{3} \mathrm{O}_{5} \mathrm{~S}[\mathrm{M}+\mathrm{H}]^{+}$396.1029, found 396.1023.

间硝基苯基( $N^{3}$-替加氟)正丙基硫醚(5e)：产率 92\%， 黄色油状液体. ${ }^{1} \mathrm{H}$ NMR (400 $\left.\mathrm{MHz}, \mathrm{CDCl}_{3}\right) \delta: 8.11$ (s, $1 \mathrm{H}), 8.00(\mathrm{~d}, J=8.0 \mathrm{~Hz}, 1 \mathrm{H}), 7.62(\mathrm{~d}, J=8.0 \mathrm{~Hz}, 1 \mathrm{H})$, $7.47 \sim 7.42(\mathrm{~m}, 2 \mathrm{H}), 5.98(\mathrm{~s}, 1 \mathrm{H}), 4.27 \sim 3.97(\mathrm{~m}, 4 \mathrm{H})$, $3.05(\mathrm{t}, J=7.4 \mathrm{~Hz}, 1 \mathrm{H}), 2.46 \sim 2.38(\mathrm{~m}, 1 \mathrm{H}), 2.07 \sim 1.89$ $(\mathrm{m}, 5 \mathrm{H}) ;{ }^{13} \mathrm{CNMR}\left(100 \mathrm{MHz}, \mathrm{CDCl}_{3}\right) \delta: 157.2(\mathrm{~d}, J=25.3$ $\mathrm{Hz}$ ), 149.0, 148.5, 139.9 (d, $J=232.6 \mathrm{~Hz}$ ), 139.3, 134.4, 129.6, 122.8, 121.9 (d, $J=33.8 \mathrm{~Hz}), 120.6,88.1,70.4$, 40.6, 33.0, 30.8, 29.7, 26.8, 23.8; TOF-HRMS (ESI) calcd for $\mathrm{C}_{17} \mathrm{H}_{19} \mathrm{FN}_{3} \mathrm{O}_{5} \mathrm{~S}[\mathrm{M}+\mathrm{H}]^{+}$396.1029, found 396.1020.

邻硝基苯基( $N^{3}$-替加氟)正丙基硫醚(5f)：产率 89\%, 
黄色油状液体. ${ }^{1} \mathrm{H}$ NMR $\left(400 \mathrm{MHz}, \mathrm{CDCl}_{3}\right) \delta: 8.18(\mathrm{~d}$, $J=8.4 \mathrm{~Hz}, 1 \mathrm{H}), 7.42 \sim 7.19(\mathrm{~m}, 4 \mathrm{H}), 5.99$ (s, 1H), 4.25 $3.97(\mathrm{~m}, 4 \mathrm{H}), 3.02(\mathrm{t}, J=7.2 \mathrm{~Hz}, 2 \mathrm{H}), 2.22 \sim 1.88(\mathrm{~m}, 6 \mathrm{H})$; ${ }^{13} \mathrm{C}$ NMR $\left(100 \mathrm{MHz}, \mathrm{CDCl}_{3}\right) \delta: 157.2(\mathrm{~d}, J=25.2 \mathrm{~Hz})$, 149.0, 146.0, 139.4 (d, $J=232.4 \mathrm{~Hz}), 137.3,133.6,126.7$, 126.1, 124.6, 122.0 (d, $J=33.7 \mathrm{~Hz}$ ), 88.2, 70.4, 40.9, 36.5, 31.4, 29.7, 26.0, 23.8; TOF-HRMS (ESI) calcd for $\mathrm{C}_{17} \mathrm{H}_{19} \mathrm{~F}-\mathrm{N}_{3} \mathrm{O}_{5} \mathrm{~S}[\mathrm{M}+\mathrm{H}]^{+} \quad 396.1029$, found 396.1022 .

对甲基苯基( $N^{3}$-替加氟)正丙基硫醚 $(\mathbf{5 g}$ )：产率 $88 \%$, 无色油状液体. ${ }^{1} \mathrm{H}$ NMR (400 MHz, $\left.\mathrm{CDCl}_{3}\right) \delta: 7.40(\mathrm{~d}$, $J=6.0 \mathrm{~Hz}, 1 \mathrm{H}), 7.28$ (d, $J=8.4 \mathrm{~Hz}, 2 \mathrm{H}), 7.10$ (d, $J=8.4$ $\mathrm{Hz}, 2 \mathrm{H}), 6.00 \sim 5.98(\mathrm{~m}, 1 \mathrm{H}), 4.26 \sim 4.21(\mathrm{~m}, 1 \mathrm{H}), 4.10 \sim$ $3.97(\mathrm{~m}, 3 \mathrm{H}), 2.97(\mathrm{~s}, 3 \mathrm{H}), 2.91(\mathrm{t}, J=7.4 \mathrm{~Hz}, 2 \mathrm{H}), 2.46 \sim$ $2.37(\mathrm{~m}, 1 \mathrm{H}), 2.08 \sim 1.92(\mathrm{~m}, 5 \mathrm{H}) ;{ }^{13} \mathrm{C}$ NMR $(100 \mathrm{MHz}$, $\left.\mathrm{CDCl}_{3}\right) \delta: 157.1(\mathrm{~d}, J=25.4 \mathrm{~Hz}), 149.0,139.9(\mathrm{~d}, J=232.5$ $\mathrm{Hz}), 136.4,132.1,130.7,129.7,122.1(\mathrm{~d}, J=33.7 \mathrm{~Hz})$, 88.0, 70.2, 40.8, 32.9, 32.3, 27.2, 23.7, 21.0; TOF-HRMS (ESI) calcd for $\mathrm{C}_{18} \mathrm{H}_{22} \mathrm{FN}_{2} \mathrm{O}_{3} \mathrm{~S}[\mathrm{M}+\mathrm{H}]^{+} 365.1335$, found 365.1332 .

对羟基苯基 ( $N^{3}$-替加氟)正丙基硫梄(5h)：产率 85\%, 无色油状液体. ${ }^{1} \mathrm{H}$ NMR $\left(400 \mathrm{MHz}, \mathrm{CDCl}_{3}\right) \delta: 7.42$ (d, $J=6.0 \mathrm{~Hz}, 1 \mathrm{H}), 7.29$ (d, $J=8.8 \mathrm{~Hz}, 1 \mathrm{H}), 6.81$ (d, $J=8.8$ $\mathrm{Hz}, 2 \mathrm{H}), 5.98(\mathrm{~d}, J=4.8 \mathrm{~Hz}, 1 \mathrm{H}), 4.25 \sim 4.20(\mathrm{~m}, 1 \mathrm{H})$, $4.05(\mathrm{t}, J=7.2 \mathrm{~Hz}, 2 \mathrm{H}), 4.02 \sim 3.95(\mathrm{~m}, 1 \mathrm{H}), 2.80(\mathrm{t}, J=$ $7.2 \mathrm{~Hz}, 2 \mathrm{H}), 2.61(\mathrm{~s}, 1 \mathrm{H}), 2.44 \sim 2.35(\mathrm{~m}, 1 \mathrm{H}), 2.05 \sim 1.73$ $(\mathrm{m}, 5 \mathrm{H}) ;{ }^{13} \mathrm{C}$ NMR $\left(100 \mathrm{MHz}, \mathrm{CDCl}_{3}\right) \delta: 157.2(\mathrm{~d}, J=25.2$ $\mathrm{Hz}), 149.0,139.9$ (d, $J=232.0 \mathrm{~Hz}), 134.3,124.3,121.9$ (d, $J=33.8 \mathrm{~Hz}), 116.2,88.1,70.3,40.8,36.7,31.6,27.1,23.7$; TOF- HRMS (ESI) calcd for $\mathrm{C}_{17} \mathrm{H}_{19} \mathrm{FN}_{2} \mathrm{O}_{4} \mathrm{~S}[\mathrm{M}+\mathrm{H}]^{+}$ 366.1050, found 366.1046 .

对氨基苯基( $N^{3}$-替加氟)正丙基硫醚(5i)：产率 84\%, 黄色油状液体. ${ }^{1} \mathrm{H}$ NMR $\left(400 \mathrm{MHz}, \mathrm{CDCl}_{3}\right) \delta: 7.38(\mathrm{~d}$, $J=6.0 \mathrm{~Hz}, 1 \mathrm{H}), 7.27(\mathrm{t}, J=8.4 \mathrm{~Hz}, 2 \mathrm{H}), 6.61(\mathrm{~d}, J=8.8$ $\mathrm{Hz}, 2 \mathrm{H}), 5.98(\mathrm{~d}, J=4.8 \mathrm{~Hz}, 1 \mathrm{H}), 4.25 \sim 4.20(\mathrm{~m}, 1 \mathrm{H})$, $4.09 \sim 3.96(\mathrm{~m}, 3 \mathrm{H}), 2.79(\mathrm{t}, J=6.4 \mathrm{~Hz}, 2 \mathrm{H}), 2.06 \sim 1.78$ $(\mathrm{m}, 5 \mathrm{H}), 1.63(\mathrm{~s}, 2 \mathrm{H}) ;{ }^{13} \mathrm{C}$ NMR $\left(100 \mathrm{MHz}, \mathrm{CDCl}_{3}\right) \delta$ : $157.2(\mathrm{~d}, J=25.4 \mathrm{~Hz}), 149.0,146.1,139.7$ (d, $J=232.2$ $\mathrm{Hz}), 134.6,122.8,121.5$ (d, $J=33.8 \mathrm{~Hz}), 115.6,88.1,70.3$, 40.8, 34.2, 33.0, 27.2, 23.8; TOF-HRMS (ESI) calcd for $\mathrm{C}_{17} \mathrm{H}_{20} \mathrm{FN}_{3} \mathrm{O}_{3} \mathrm{~S} \quad[\mathrm{M}+\mathrm{H}]^{+}$365.1209, found 365.1214.

邻苯甲酰氨基苯基( $N^{3}$-替加氟)正丙基硫醚 $(\mathbf{5} \mathbf{j})$ : 产 率 90\%, 白色固体; m.p. 96 97 ${ }^{\circ} \mathrm{C} ;{ }^{1} \mathrm{H}$ NMR $(400 \mathrm{MHz}$, $\left.\mathrm{CDCl}_{3}\right) \delta: 9.44(\mathrm{~s}, 1 \mathrm{H}), 8.56(\mathrm{~d}, J=7.6 \mathrm{~Hz}, 1 \mathrm{H}), 7.95(\mathrm{~d}$, $J=8.4 \mathrm{~Hz}, 1 \mathrm{H}), 7.61 \sim 7.50(\mathrm{~m}, 4 \mathrm{H}), 7.37(\mathrm{~d}, J=7.6 \mathrm{~Hz}$, $2 \mathrm{H}), 7.09(\mathrm{t}, J=7.6 \mathrm{~Hz}, 1 \mathrm{H}), 5.93 \sim 5.91(\mathrm{~m}, 1 \mathrm{H}), 4.21 \sim$ $4.17(\mathrm{~m}, 1 \mathrm{H}), 4.06 \sim 3.99(\mathrm{~m}, 3 \mathrm{H}), 2.82(\mathrm{t}, J=7.2 \mathrm{~Hz}, 2 \mathrm{H})$, $2.40 \sim 2.30(\mathrm{~m}, 1 \mathrm{H}), 2.06 \sim 1.82(\mathrm{~m}, 5 \mathrm{H}) ;{ }^{13} \mathrm{C}$ NMR $(100$ $\left.\mathrm{MHz}, \mathrm{CDCl}_{3}\right): \delta 157.0(\mathrm{~d}, J=25.2 \mathrm{~Hz}), 149.0,139.9$ (d, $J=232.6 \mathrm{~Hz}), 139.6,135.6,134.8,132.0,129.9,128.9$, 127.1, 124.3, 122.8, 121.8 (d, $J=33.7 \mathrm{~Hz}), 120.4,88.0$, 70.3, 40.5, 33.7, 33.0, 27.4, 23.8; TOF-HRMS (ESI) calcd for $\mathrm{C}_{24} \mathrm{H}_{24} \mathrm{FN}_{3} \mathrm{O}_{4} \mathrm{~S}[\mathrm{M}+\mathrm{H}]^{+}$469.1472, found 469.1476.

1,2,3-三唑基 ( $N^{3}$ - 替加氟)正丙基硫醚 (5k): 产率 $91 \%$, 无色油状液体. ${ }^{1} \mathrm{H}$ NMR ( $400 \mathrm{MHz}, \mathrm{CDCl}_{3}$ ) $\delta: 8.02$ (s, 1H), $7.42(\mathrm{~d}, J=5.6 \mathrm{~Hz}, 1 \mathrm{H}), 6.02 \sim 5.97(\mathrm{~m}, 1 \mathrm{H})$, $4.27 \sim 3.97(\mathrm{~m}, 4 \mathrm{H}), 2.47 \sim 2.38(\mathrm{~m}, 1 \mathrm{H}), 2.10 \sim 1.87(\mathrm{~m}$, $5 \mathrm{H}) ;{ }^{13} \mathrm{C}$ NMR $\left(100 \mathrm{MHz}, \mathrm{CDCl}_{3}\right) \delta: 157.2(\mathrm{~d}, J=25.2$ $\mathrm{Hz}), 149.0,139.5$ (d, $J=232.4 \mathrm{~Hz}), 136.6,121.9$ (d, $J=$ $33.7 \mathrm{~Hz}), 88.1,70.3,40.7,40.4,36.6,31.2,27.1,23.8$; TOF-HRMS (ESI) calcd for $\mathrm{C}_{13} \mathrm{H}_{16} \mathrm{FN}_{5} \mathrm{O}_{3} \mathrm{~S}[\mathrm{M}+\mathrm{H}]^{+}$ 341.0958, found 341.0963.

2 -糠基( $N^{3}$-替加氟)正丙基硫醚(5I): 产率 93\%, 无色 油状液体. ${ }^{1} \mathrm{H}$ NMR $\left(400 \mathrm{MHz}, \mathrm{CDCl}_{3}\right) \delta: 7.77$ (d, $J=8.0$ $\mathrm{Hz}, 2 \mathrm{H}), 7.40$ (d, $J=8.0 \mathrm{~Hz}, 1 \mathrm{H}), 7.34$ (d, $J=8.0 \mathrm{~Hz}, 2 \mathrm{H})$, $5.97 \sim 5.95(\mathrm{~m}, 1 \mathrm{H}), 4.25 \sim 4.20(\mathrm{~m}, 1 \mathrm{H}), 4.09(\mathrm{t}, J=6.2$ $\mathrm{Hz}, 2 \mathrm{H}), 4.03 \sim 3.97(\mathrm{~m}, 3 \mathrm{H}), 2.45 \sim 2.36(\mathrm{~m}, 3 \mathrm{H}), 2.10 \sim$ $1.89(\mathrm{~m}, 5 \mathrm{H}) ;{ }^{13} \mathrm{C}$ NMR $\left(100 \mathrm{MHz}, \mathrm{CDCl}_{3}\right) \delta: 157.1(\mathrm{~d}$, $J=25.4 \mathrm{~Hz}), 148.9,144.9,139.8$ (d, $J=232.2 \mathrm{~Hz}), 132.8$, 129.9, 127.9, 121.9 (d, $J=33.8 \mathrm{~Hz}), 88.2,70.4,68.5,38.5$, 33.0, 27.0, 23.7, 21.6; TOF-HRMS (ESI) calcd for $\mathrm{C}_{16} \mathrm{H}_{20}{ }^{-}$ $\mathrm{FN}_{2} \mathrm{O}_{4} \mathrm{~S}[\mathrm{M}+\mathrm{H}]^{+} 355.1128$, found 355.1122.

2-塞吩基( $N^{3}$-替加氟)正丙基硫醚(5m): 产率 $90 \%$, 无色油状液体. ${ }^{1} \mathrm{H}$ NMR $\left(400 \mathrm{MHz}, \mathrm{CDCl}_{3}\right) \delta: 7.39(\mathrm{~d}$, $J=5.6 \mathrm{~Hz}, 1 \mathrm{H}), 7.33(\mathrm{~d}, J=5.2 \mathrm{~Hz}, 1 \mathrm{H}), 7.17(\mathrm{~d}, J=3.6$ $\mathrm{Hz}, 1 \mathrm{H}), 6.98 \sim 6.96(\mathrm{~m}, 1 \mathrm{H}), 5.99(\mathrm{~d}, J=4.8 \mathrm{~Hz}, 1 \mathrm{H})$, $4.25 \sim 4.20(\mathrm{~m}, 1 \mathrm{H}), 4.08 \sim 3.96(\mathrm{~m}, 3 \mathrm{H}), 2.81(\mathrm{t}, J=7.2$ $\mathrm{Hz}, 2 \mathrm{H}), 2.44-2.37(\mathrm{~m}, 1 \mathrm{H}), 2.10 \sim 1.88(\mathrm{~m}, 5 \mathrm{H}) ;{ }^{13} \mathrm{C} \mathrm{NMR}$ $\left(100 \mathrm{MHz}, \mathrm{CDCl}_{3}\right) \delta: 157.0(\mathrm{~d}, J=25.3 \mathrm{~Hz}), 149.0,139.9$ (d, $J=232.5 \mathrm{~Hz}), 134.4,133.7,129.6,127.6,121.7$ (d, $J=$ $33.8 \mathrm{~Hz}), 88.1,70.3,40.5,36.4,33.0,27.1,23.8$; TOFHRMS (ESI) calcd for $\mathrm{C}_{15} \mathrm{H}_{18} \mathrm{FN}_{2} \mathrm{O}_{3} \mathrm{~S}_{2} \quad[\mathrm{M}+\mathrm{H}]{ }^{+}$ 357.0743, found 357.0749.

2-吡啶基( $N^{3}$-替加氟)正丙基硫醚(5n): 产率 83\%, 无色油状液体. ${ }^{1} \mathrm{H}$ NMR $\left(400 \mathrm{MHz}, \mathrm{CDCl}_{3}\right) \delta: 8.39(\mathrm{~d}$, $J=3.2 \mathrm{~Hz}, 1 \mathrm{H}), 7.48 \sim 7.43(\mathrm{~m}, 1 \mathrm{H}), 7.40(\mathrm{~d}, J=6.0 \mathrm{~Hz}$, $1 \mathrm{H}), 7.15(\mathrm{~d}, J=8.4 \mathrm{~Hz}, 1 \mathrm{H}), 6.00 \sim 5.98(\mathrm{~m}, 1 \mathrm{H}), 4.26 \sim$ $4.20(\mathrm{~m}, 1 \mathrm{H}), 4.13 \sim 4.08(\mathrm{~m}, 2 \mathrm{H}), 4.02 \sim 3.97(\mathrm{~m}, 1 \mathrm{H})$, $3.21(\mathrm{t}, J=7.2 \mathrm{~Hz}, 2 \mathrm{H}), 2.44 \sim 2.35(\mathrm{~m}, 1 \mathrm{H}), 2.11 \sim 2.01$ $(\mathrm{m}, 4 \mathrm{H}), 1.96 \sim 1.86(\mathrm{~m}, 1 \mathrm{H}) ;{ }^{13} \mathrm{C}$ NMR $(100 \mathrm{MHz}$, $\left.\mathrm{CDCl}_{3}\right): \delta 158.6,157.0(\mathrm{~d}, J=25.1 \mathrm{~Hz}), 149.4,149.0$, 
$139.9(\mathrm{~d}, J=232.4 \mathrm{~Hz}), 135.9,122.3,121.6$ (d, $J=33.7$ Hz), 88.1, 70.3, 40.9, 33.0, 27.6, 27.3, 23.8; TOF-HRMS (ESI) calcd for $\mathrm{C}_{16} \mathrm{H}_{19} \mathrm{~F}-\mathrm{N}_{3} \mathrm{O}_{3} \mathrm{~S}[\mathrm{M}+\mathrm{H}]^{+}$352.1131, found 352.1127.

苯基 ( $N^{3}$-替加氟)乙基硫醚 $(\mathbf{5 0})$ : 产率 $94 \%$, 无色油 状液体. ${ }^{1} \mathrm{H}$ NMR $\left(400 \mathrm{MHz}, \mathrm{CDCl}_{3}\right) \delta: 7.78(\mathrm{~d}, J=8.4$ $\mathrm{Hz}, 2 \mathrm{H}), 7.43(\mathrm{~d}, J=7.2 \mathrm{~Hz}, 2 \mathrm{H}), 7.34 \sim 7.27(\mathrm{~m}, 3 \mathrm{H})$, $7.18(\mathrm{t}, J=7.4 \mathrm{~Hz}, 1 \mathrm{H}), 5.97 \sim 5.95(\mathrm{~m}, 1 \mathrm{H}), 4.23 \sim 4.19$ $(\mathrm{m}, 3 \mathrm{H}), 4.00 \sim 3.96(\mathrm{~m}, 1 \mathrm{H}), 3.21 \sim 3.18(\mathrm{~m}, 2 \mathrm{H}), 2.46 \sim$ $2.35(\mathrm{~m}, 1 \mathrm{H}), 2.07 \sim 2.00(\mathrm{~m}, 2 \mathrm{H}), 1.96 \sim 1.87(\mathrm{~m}, 1 \mathrm{H})$; ${ }^{13} \mathrm{C}$ NMR $\left(100 \mathrm{MHz}, \mathrm{CDCl}_{3}\right) \delta: 157.0(\mathrm{~d}, J=25.4 \mathrm{~Hz})$, 148.9, 139.8 (d, $J=232.9 \mathrm{~Hz}), 135.3,129.0,128.7,126.1$, 121.8 (d, $J=33.7 \mathrm{~Hz}), 88.1,70.4,41.2,33.0,29.8,23.8$; TOF-HRMS (ESI) calcd for $\mathrm{C}_{16} \mathrm{H}_{17} \mathrm{FN}_{2} \mathrm{O}_{3} \mathrm{~S}[\mathrm{M}+\mathrm{H}]^{+}$ 336.0944, found 336.0950.

苯基 $\left(N^{3}\right.$-替加氟)正丁基硫醚(5p): 产率 91\%, 无色 油状液体. ${ }^{1} \mathrm{H}$ NMR $\left(400 \mathrm{MHz}, \mathrm{CDCl}_{3}\right) \delta: 7.65 \sim 7.62(\mathrm{~m}$, $1 \mathrm{H}), 7.55 \sim 7.52(\mathrm{~m}, 1 \mathrm{H}), 7.41 \sim 7.38(\mathrm{~m}, 1 \mathrm{H}), 7.35 \sim 7.30$ (m, $2 \mathrm{H}), 7.21 \sim 7.16(\mathrm{~m}, 1 \mathrm{H}), 6.01 \sim 5.99(\mathrm{~m}, 1 \mathrm{H}), 4.27 \sim$ $4.21(\mathrm{~m}, 1 \mathrm{H}), 4.04 \sim 3.93(\mathrm{~m}, 3 \mathrm{H}), 2.97(\mathrm{t}, J=7.2 \mathrm{~Hz}, 2 \mathrm{H})$, $2.48 \sim 2.35(\mathrm{~m}, 1 \mathrm{H}), 2.11 \sim 2.02(\mathrm{~m}, 2 \mathrm{H}), 1.82 \sim 1.77(\mathrm{~m}$, $3 \mathrm{H}), 1.73 \sim 1.65(\mathrm{~m}, 2 \mathrm{H}) ;{ }^{13} \mathrm{C} \mathrm{NMR}\left(100 \mathrm{MHz}, \mathrm{CDCl}_{3}\right) \delta$ : $157.5(\mathrm{~d}, J=25.1 \mathrm{~Hz}), 149.5,139.3(\mathrm{~d}, J=232.5 \mathrm{~Hz})$, $137.2,129.4,129.3,126.2,121.2$ (d, $J=33.8 \mathrm{~Hz}), 88.5$, 70.8, 42.0, 33.5, 29.2, 27.5, 26.5, 24.3; TOF-HRMS (ESI) calcd for $\mathrm{C}_{18} \mathrm{H}_{22} \mathrm{FN}_{2} \mathrm{O}_{3} \mathrm{~S}[\mathrm{M}+\mathrm{H}]^{+} 365.1335$, found 365.1340 .

苯基( $N^{3}$-替加氟)正戊基硫醚(5q): 产率 92\%, 无色 油状液体. ${ }^{1} \mathrm{H}$ NMR $\left(400 \mathrm{MHz}, \mathrm{CDCl}_{3}\right) \delta: 7.38(\mathrm{~d}, J=6.0$ $\mathrm{Hz}, 1 \mathrm{H}), 7.32 \sim 7.25(\mathrm{~m}, 4 \mathrm{H}), 7.17 \sim 7.13(\mathrm{~m}, 1 \mathrm{H}), 6.00 \sim$ $5.98(\mathrm{~m}, 1 \mathrm{H}), 4.22 \sim 4.20(\mathrm{~m}, 1 \mathrm{H}), 4.02 \sim 3.89(\mathrm{~m}, 3 \mathrm{H})$, $2.91(\mathrm{t}, J=7.4 \mathrm{~Hz}, 2 \mathrm{H}), 2.45 \sim 2.36(\mathrm{~m}, 1 \mathrm{H}), 2.09 \sim 2.00$ $(\mathrm{m}, 2 \mathrm{H}), 1.95 \sim 1.88(\mathrm{~m}, 1 \mathrm{H}), 1.72 \sim 1.63(\mathrm{~m}, 4 \mathrm{H}), 1.52 \sim$ $1.44(\mathrm{~m}, 2 \mathrm{H}) ;{ }^{13} \mathrm{C}$ NMR $\left(100 \mathrm{MHz}, \mathrm{CDCl}_{3}\right) \delta: 157.1(\mathrm{~d}$, $J=25.1 \mathrm{~Hz}), 149.0,139.9$ (d, $J=232.2 \mathrm{~Hz}), 136.7,128.9$, 128.8, 125.7, 121.5 (d, $J=33.8 \mathrm{~Hz}), 88.0,70.3,41.5,33.3$, 33.0, 28.7, 27.0, 26.0, 23.8; TOF-HRMS (ESI) calcd for $\mathrm{C}_{19} \mathrm{H}_{23} \mathrm{FN}_{2} \mathrm{O}_{3} \mathrm{~S}[\mathrm{M}+\mathrm{H}]^{+}$378.1413, found 378.1418.

苯基 ( $N^{3}$-替加氟)正己基硫醚(5r): 产率 91\%, 无色 油状液体. ${ }^{1} \mathrm{H}$ NMR $\left(400 \mathrm{MHz}, \mathrm{CDCl}_{3}\right) \delta: 7.30(\mathrm{~d}, J=6.0$ $\mathrm{Hz}, 1 \mathrm{H}), 7.25 \sim 7.17(\mathrm{~m}, 4 \mathrm{H}), 7.10 \sim 7.06(\mathrm{~m}, 1 \mathrm{H}), 5.92 \sim$ $5.90(\mathrm{~m}, 1 \mathrm{H}), 4.17 \sim 4.11(\mathrm{~m}, 1 \mathrm{H}), 3.94 \sim 3.81(\mathrm{~m}, 3 \mathrm{H})$, $2.83(\mathrm{t}, J=7.2 \mathrm{~Hz}, 2 \mathrm{H}), 2.37 \sim 2.29(\mathrm{~m}, 1 \mathrm{H}), 2.00 \sim 1.93$ $(\mathrm{m}, 2 \mathrm{H}), 1.85 \sim 1.78(\mathrm{~m}, 1 \mathrm{H}), 1.61 \sim 1.51(\mathrm{~m}, 4 \mathrm{H}), 1.43 \sim$ $1.35(\mathrm{~m}, 2 \mathrm{H}), 1.31 \sim 1.25(\mathrm{~m}, 2 \mathrm{H}) ;{ }^{13} \mathrm{CNMR}(100 \mathrm{MHz}$,
$\left.\mathrm{CDCl}_{3}\right) \delta: 157.1(\mathrm{~d}, J=25.1 \mathrm{~Hz}), 149.0,139.9(\mathrm{~d}, J=232.2$ Hz), 136.9, 129.1, 128.9, 128.8, 127.5, 127.2, 125.7, 121.6 (d, $J=33.8 \mathrm{~Hz}$ ), 88.0, 70.3, 41.6, 33.4, 33.0, 29.0, 28.4, 27.4, 26.4, 23.8; TOF-HRMS (ESI) calcd for $\mathrm{C}_{20} \mathrm{H}_{25} \mathrm{~F}-$ $\mathrm{N}_{2} \mathrm{O}_{3} \mathrm{~S}[\mathrm{M}+\mathrm{H}]^{+} 392.1570$, found 392.1576 .

1,2,3-三唑基( $N^{3}$-替加氟)正已基硫醚(5s)：产率 $91 \%$, 无色油状液体. ${ }^{1} \mathrm{H}$ NMR $\left(400 \mathrm{MHz}, \mathrm{CDCl}_{3}\right) \delta: 7.57(\mathrm{t}$, $J=28.6 \mathrm{~Hz}, 1 \mathrm{H}), 7.43 \sim 7.39(\mathrm{~m}, 1 \mathrm{H}), 6.04 \sim 6.00(\mathrm{~m}$, $1 \mathrm{H}), 4.28 \sim 4.21(\mathrm{~m}, 1 \mathrm{H}), 4.05 \sim 3.90(\mathrm{~m}, 3 \mathrm{H}), 2.97 \sim$ $2.86(\mathrm{~m}, 2 \mathrm{H}), 2.49 \sim 2.39(\mathrm{~m}, 1 \mathrm{H}), 2.11 \sim 2.03(\mathrm{~m}, 2 \mathrm{H})$, $2.00 \sim 1.90(\mathrm{~m}, 2 \mathrm{H}), 1.70 \sim 1.60(\mathrm{~m}, 4 \mathrm{H}), 1.53 \sim 1.40$ $(\mathrm{m}, 2 \mathrm{H}), 1.39 \sim 1.33(\mathrm{~m}, 2 \mathrm{H}) ;{ }^{13} \mathrm{CNMR}(100 \mathrm{MHz}$, $\left.\mathrm{CDCl}_{3}\right) \delta: 157.0(\mathrm{~d}, J=24.7 \mathrm{~Hz}), 149.0,140.4(\mathrm{~d}, J=$ $232.9 \mathrm{~Hz}), 136.2,121.5$ (d, $J=33.5 \mathrm{~Hz}), 88.1,70.2$, $41.6, \quad 36.5, \quad 34.6, \quad 33.0, \quad 29.2, \quad 27.6, \quad 25.8, \quad 23.8$; TOF-HRMS (ESI) calcd for $\mathrm{C}_{16} \mathrm{H}_{23} \mathrm{FN}_{5} \mathrm{O}_{3} \mathrm{~S}[\mathrm{M}+\mathrm{H}]^{+}$ 384.1506, found 384.1512 .

苯基 $\left(N^{3}\right.$-替加氟)正丙基硒醚 $(\mathbf{5 t})$ : 产率 $90 \%$, 淡黄 色油状液体. ${ }^{1} \mathrm{H}$ NMR $\left(400 \mathrm{MHz}, \mathrm{CDCl}_{3}\right) \delta: 7.55$ (d, $J=$ $8.0 \mathrm{~Hz}, 2 \mathrm{H}), 7.42(\mathrm{~d}, J=5.6 \mathrm{~Hz}, 1 \mathrm{H}), 7.31 \sim 7.28(\mathrm{~m}, 3 \mathrm{H})$, $6.03 \sim 5.97(\mathrm{~m}, 1 \mathrm{H}), 4.28 \sim 4.23(\mathrm{~m}, 1 \mathrm{H}), 4.10(\mathrm{t}, J=7.0$ $\mathrm{Hz}, 2 \mathrm{H}), 4.06 \sim 4.00(\mathrm{~m}, 1 \mathrm{H}), 2.95(\mathrm{t}, J=7.4 \mathrm{~Hz}, 2 \mathrm{H})$, $2.48 \sim 2.40(\mathrm{~m}, 1 \mathrm{H}), 2.12 \sim 1.92(\mathrm{~m}, 5 \mathrm{H}) ;{ }^{13} \mathrm{C}$ NMR $(100$ $\left.\mathrm{MHz}, \mathrm{CDCl}_{3}\right) \delta: 157.1(\mathrm{~d}, J=25.3 \mathrm{~Hz}), 149.0,139.9$ (d, $J=232.5 \mathrm{~Hz}), 133.0,129.1,127.0,122.0(\mathrm{~d}, J=33.8 \mathrm{~Hz})$, 88.1, 70.3, 41.6, 33.0, 28.1, 24.9 23.8; TOF-HRMS (ESI) calcd for $\mathrm{C}_{17} \mathrm{H}_{20} \mathrm{FN}_{2} \mathrm{O}_{3} \mathrm{Se}[\mathrm{M}+\mathrm{H}]^{+}$399.0623, found 399.0627 .

对甲基苯基( $\mathrm{N}^{3}$-替加氟)正丙基硒梄 $(5 \mathbf{u})$ ：产率 86\%， 无色油状液体. ${ }^{1} \mathrm{H}$ NMR (400 MHz, $\mathrm{CDCl}_{3}$ ) $\delta: 7.45 \sim 7.39$ (m, 3H), $7.08(\mathrm{~d}, J=8.0 \mathrm{~Hz}, 2 \mathrm{H}), 6.00 \sim 5.98(\mathrm{~m}, 1 \mathrm{H})$, $4.27 \sim 4.21(\mathrm{~m}, 1 \mathrm{H}), 4.09 \sim 3.98(\mathrm{~m}, 3 \mathrm{H}), 2.87(\mathrm{t}, J=7.4$ $\mathrm{Hz}, 3 \mathrm{H}), 2.45 \sim 2.38(\mathrm{~m}, 1 \mathrm{H}), 2.33(\mathrm{~s}, 3 \mathrm{H}), 2.05 \sim 1.87(\mathrm{~m}$, $5 \mathrm{H}) ;{ }^{13} \mathrm{C}$ NMR $\left(100 \mathrm{MHz}, \mathrm{CDCl}_{3}\right) \delta: 157.1$ (d, $J=23.1$ $\mathrm{Hz}), 149.0,139.9$ (d, $J=234.4 \mathrm{~Hz}), 137.2,133.6,129.9$, 125.9, 121.6 (d, $J=34.1 \mathrm{~Hz}), 88.1,70.3,41.6,33.0,28.0$, 25.2, 23.8, 21.1; TOF-HRMS (ESI) calcd for $\mathrm{C}_{18} \mathrm{H}_{22} \mathrm{~F}-$ $\mathrm{N}_{2} \mathrm{O}_{3} \mathrm{Se}[\mathrm{M}+\mathrm{H}]^{+}$413.0780, found 413.0784.

对甲氧基苯基 ( $N^{3}$-替加氟)正丙基硒醚 (5v)：产率 $80 \%$, 无色油状液体. ${ }^{1} \mathrm{H}$ NMR $\left(400 \mathrm{MHz}, \mathrm{CDCl}_{3}\right) \delta: 7.50$ (d, $J=8.8 \mathrm{~Hz}, 2 \mathrm{H}), 7.39$ (d, $J=5.6 \mathrm{~Hz}, 1 \mathrm{H}), 6.82(\mathrm{~d}, J=$ $8.8 \mathrm{~Hz}, 2 \mathrm{H}), 6.00 \sim 5.97(\mathrm{~m}, 1 \mathrm{H}), 4.26 \sim 4.20(\mathrm{~m}, 1 \mathrm{H})$, $4.09 \sim 3.97(\mathrm{~m}, 3 \mathrm{H}), 3.80(\mathrm{~s}, 3 \mathrm{H}), 2.85 \sim 2.79(\mathrm{~m}, 2 \mathrm{H})$, $2.47 \sim 2.37(\mathrm{~m}, 1 \mathrm{H}), 2.10 \sim 1.88(\mathrm{~m}, 5 \mathrm{H}) ;{ }^{13} \mathrm{C}$ NMR $(100$ $\left.\mathrm{MHz}, \mathrm{CDCl}_{3}\right) \delta: 159.4,157.1(\mathrm{~d}, J=25.1 \mathrm{~Hz}), 149.0$, 
$139.9(\mathrm{~d}, J=232.4 \mathrm{~Hz}), 136.1,121.6(\mathrm{~d}, J=33.8 \mathrm{~Hz})$, 119.5, 114.8, 88.0, 70.3, 55.3, 41.6, 33.0, 27.9, 26.0, 23.8; TOF-HRMS (ESI) calcd for $\mathrm{C}_{18} \mathrm{H}_{22} \mathrm{FN}_{2} \mathrm{O}_{4} \mathrm{Se}[\mathrm{M}+\mathrm{H}]^{+}$ 429.0729, found 429.0735 .

对氧基苯基 $\left(N^{3}\right.$ - 替加氟) 正丙基硒醚 $(\mathbf{5 w})$ : 产率 $95 \%$, 无色油状液体. ${ }^{1} \mathrm{H} \mathrm{NMR}\left(400 \mathrm{MHz}, \mathrm{CDCl}_{3}\right) \delta: 7.51$ $(\mathrm{s}, 3 \mathrm{H}), 7.41(\mathrm{~d}, J=5.6 \mathrm{~Hz}, 1 \mathrm{H}), 6.00 \sim 5.97(\mathrm{~m}, 1 \mathrm{H})$, $4.27 \sim 4.21(\mathrm{~m}, 1 \mathrm{H}), 4.11 \sim 3.97(\mathrm{~m}, 3 \mathrm{H}), 3.02 \sim 2.96(\mathrm{~m}$, $2 \mathrm{H}), 2.45 \sim 2.39(\mathrm{~m}, 1 \mathrm{H}), 2.12 \sim 2.02(\mathrm{~m}, 4 \mathrm{H}), 1.96 \sim 1.91$ $(\mathrm{m}, 1 \mathrm{H}) ;{ }^{13} \mathrm{C}$ NMR $\left(100 \mathrm{MHz}, \mathrm{CDCl}_{3}\right) \delta: 157.1$ (d, $J=25.1$ $\mathrm{Hz}), 149.0,139.9$ (d, $J=232.9 \mathrm{~Hz}), 138.4,132.3,131.2$, 129.9, 127.9, 121.9 (d, $J=33.8 \mathrm{~Hz}), 118.8,109.7$, 88.1, 70.4, 41.4, 33.0. 27.7, 24.1, 23.8; TOF-HRMS (ESI) calcd for $\mathrm{C}_{18} \mathrm{H}_{19} \mathrm{FN}_{3} \mathrm{O}_{3} \mathrm{Se}[\mathrm{M}+\mathrm{H}]^{+}$424.0576, found 424.0571 .

对氯苯基 ( $N^{3}$-替加氟)正丙基硒醚 $(\mathbf{5 x})$ ：产率 93\%, 无色油状液体. ${ }^{1} \mathrm{H}$ NMR (400 $\left.\mathrm{MHz}, \mathrm{CDCl}_{3}\right) \delta: 7.44$ (d, $J=8.4 \mathrm{~Hz}, 2 \mathrm{H}), 7.40$ (d, $J=5.6 \mathrm{~Hz}, 1 \mathrm{H}), 7.22$ (d, $J=8.4$ $\mathrm{Hz}, 2 \mathrm{H}), 5.99 \sim 5.96(\mathrm{~m}, 1 \mathrm{H}), 4.25 \sim 4.20(\mathrm{~m}, 1 \mathrm{H}), 4.08 \sim$ $3.96(\mathrm{~m}, 3 \mathrm{H}), 2.88(\mathrm{t}, J=7.4 \mathrm{~Hz}, 3 \mathrm{H}), 2.44 \sim 2.36(\mathrm{~m}, 1 \mathrm{H})$, $2.10 \sim 1.99(\mathrm{~m}, 4 \mathrm{H}), 1.97 \sim 1.87(\mathrm{~m}, 1 \mathrm{H}) ;{ }^{13} \mathrm{C}$ NMR $(100$ $\left.\mathrm{MHz}, \mathrm{CDCl}_{3}\right) \delta: 157.1$ (d, $\left.J=25.2 \mathrm{~Hz}\right), 149.0,139.9$ (d, $J=232.5 \mathrm{~Hz}), 134.5,133.3,129.2,127.9,122.1$ (d, $J=$ $33.7 \mathrm{~Hz}), 118.7,88.0,70.3,41.5$, 33.0. 27.9, 25.3, 23.8; TOF-HRMS (ESI) calcd for $\mathrm{C}_{17} \mathrm{H}_{19} \mathrm{ClFN}_{2} \mathrm{O}_{3} \mathrm{Se}[\mathrm{M}+\mathrm{H}]^{+}$ 433.0233, found 433.0238.

苄基 $\left(N^{3}\right.$-替加氟)正丙基硒醚(5y)：产率 $81 \%$, 黄色 油状液体. ${ }^{1} \mathrm{H}$ NMR (400 MHz, $\left.\mathrm{CDCl}_{3}\right) \delta: 7.77$ (d, $J=8.0$ $\mathrm{Hz}, 1 \mathrm{H}), 7.45 \sim 7.20(\mathrm{~m}, 5 \mathrm{H}), 5.98(\mathrm{t}, J=7.2 \mathrm{~Hz}, 1 \mathrm{H})$, $4.27 \sim 4.20(\mathrm{~m}, 1 \mathrm{H}), 4.15 \sim 4.08(\mathrm{~m}, 2 \mathrm{H}), 4.04 \sim 3.97(\mathrm{~m}$, 2H), $2.72(\mathrm{t}, J=3.6 \mathrm{~Hz}, 1 \mathrm{H}), 2.45(\mathrm{~s}, 2 \mathrm{H}), 2.12 \sim 1.86(\mathrm{~m}$, $5 \mathrm{H}) ;{ }^{13} \mathrm{C}$ NMR $\left(100 \mathrm{MHz}, \mathrm{CDCl}_{3}\right) \delta: 157.1(\mathrm{~d}, J=25.3$ Hz), 149.0, 139.9 (d, $J=232.3 \mathrm{~Hz}$ ), 129.9, 129.1, 128.5, 121.9 (d, $J=33.8 \mathrm{~Hz}$ ), 88.2, 70.4, 68.5, 35.3, 33.1, 33.0, 27.0, 23.7, 21.7; TOF-HRMS (ESI) calcd for $\mathrm{C}_{18} \mathrm{H}_{21} \mathrm{~F}-$ $\mathrm{N}_{2} \mathrm{O}_{3} \mathrm{Se}[\mathrm{M}+\mathrm{H}]^{+}$412.0701, found 412.0706.

\section{3 替加氟衍生物体外抗肿瘤活性测试}

\subsubsection{CCK-8 法测试癌细胞增殖}

按细胞数每孔 5000 个, 将细胞铺入 96 孔板, 设置 空白组(无细胞)、对照组(不经处理)和实验组(药物处理) 并每组设置 3 个复孔, 细胞贴壁后弃去原有培养基, 空 白组和对照组加 $100 \mu \mathrm{L}$ 培养基, 按 20 700 $\mu \mathrm{g} / \mathrm{mL}$ 设置 浓度梯度, 将待测药物与培养基混合后加入 96 孔板实 验组孔中, 体积也为 $100 \mu \mathrm{L}$, 培养 $24 \mathrm{~h}$ 后弃去所有孔中

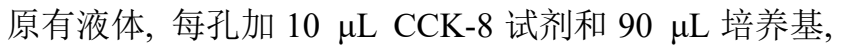
培养 $2 \sim 4 \mathrm{~h}$ 后酶标仪测吸光度，按公式计算抑制率:
细胞抑制率 $(\%)=1-[(A$ 实验组 $-A$ 空白组 $) /(A$ 对照组 $A$ 空白组 $)] \times 100 \%$

\subsubsection{DAPI 染色法观察细胞调亡}

将状态良好的细胞分为两组: (1)对照组, 贴壁后培 养箱培养 24 h. (2) 5k 组, 细胞贴壁后用 $\mathbf{5 k}(100 \mu \mathrm{g} / \mathrm{mL})$ 处理 $24 \mathrm{~h}$. 细胞铺在 $35 \mathrm{~mm}$ 培养血中, 贴壁后按分组处 理. $24 \mathrm{~h}$ 后弃去培养基, PBS 清洗三遍. 各组加入 $0.5 \mathrm{~mL}$ 的 4\%多聚甲醛, 室温固定 $15 \mathrm{~min}$. PBS 洗三次, 每次 10 min. 滴加 $0.5 \%$ 的 Triton X-100 (5 $\mu \mathrm{L}$ Triton X-100 原 液 $+995 \mu \mathrm{L}$ PBS 溶液), 室温通透 $10 \mathrm{~min}$. PBS 洗三次, 每次 $10 \mathrm{~min}$. DAPI 染剂 $0.5 \mathrm{~mL}$ 避光孵育 $10 \mathrm{~min}$. 苂光 显微镜观察, 拍照.

\subsection{3 流式细胞实验}

将 HCT116 细胞分两组铺板, 每个样品设置 3 个复 孔, (1)对照组，贴壁 $24 \mathrm{~h}$ 后进行下一步; (2) 5k $(5 \mu \mathrm{g} / \mathrm{mL}$, $30 \mu \mathrm{g} / \mathrm{mL}$ ) 处理组, 贴壁后用化合物 $\mathbf{5 k}$ 处理 $24 \mathrm{~h}$ 后进行 下一步. 细胞收集: $1.5 \mathrm{~mL}$ 胰酶充分消化两组细胞, 随 后等体积完全培养基终止消化, 将细胞悬液按 1700 转 离心 $7 \mathrm{~min}$, 弃上清. $1 \mathrm{~mL}$ 预冷的 PBS 重悬细胞, 再次 1700 转离心 $7 \mathrm{~min}$, 弃上清, 轻弹管底分散细胞避免成 才. 细胞固定: $1 \mathrm{~mL}$ PBS 重悬细胞, $3 \mathrm{~mL}$ 预冷的无水乙 醇使最终浓度为 $75 \%, 4{ }^{\circ} \mathrm{C}$ 固定 $4 \mathrm{~h}$. 按染色缓冲剂/PI (Propidium Iodide)/RNaea A (Ribonuclease A) $(V: V:$ $V=100 ： 5: 2$ )配制染色工作液, 每管样品加入 $500 \mu \mathrm{L}$ 工作液, 充分重悬, $37{ }^{\circ} \mathrm{C}$ 避光温浴 $30 \mathrm{~min}, 24 \mathrm{~h}$ 内用流 式细胞仪完成检测. 重复试验 $2 \sim 3$ 次.

辅助材料(Supporting Information) 所有化合物 ${ }^{1} \mathrm{H}$ $\mathrm{NMR}$ 和 ${ }^{13} \mathrm{C} \mathrm{NMR}$ 的光谱数据. 这些材料可以免费从本 刊网站(http://sioc-journal.cn/)上下载.

\section{References}

[1] Tsuboi, M.; Hamada, C.; Kato, H.; Ohta, M. Chemotherapy 2017, 62, 357.

[2] Ranieri, G.; Marech, I.; Porcelli, M. Giotta, F.; Palmiotti, G.; Laricchia, G.; Fazio, V.; Gadaleta, C. D. Oncotarget 2018, 9, 8197.

[3] Longleyd, B.; Harkin, D. P.; Johnston, P. G. Nat. Rev. Cancer 2003, 3,330 .

[4] Broto, M.; Mccabe, R.; Galve, R.; Marco, M. P. Analyst 2017, 142, 2404.

[5] Arias, J. L.; Sáez-Fernández, E.; López-Viota, M.; Biedma-Ortiz, R. A.; Ruiz, M. A. Med. Chem. 2012, 8, 516.

[6] Boisdron-celle, M.; Capitain, O.; Faroux, R.; Borg, C.; Metges, J. P.; Galais, M. P.; Kaassis, M.; Bennouna, J.; Bouhier-leporrier, O.; Francois, E.; Baumgaertner, I.; Guerin-Meyer, V.; Cojocarasu, O.; Roemer-Becuwe, C.; Stampfli, C.; Rosenfeld, L.; Lecompte, T.; Berger, V.; Morel, A.; Gamelin, E. Semin. Oncol. 2017, 44, 13.

[7] Zhang, Y.-X.; Dai, G.-F.; Wang, L.; Tao, J.-C. Bioorg. Med. Chem. Lett. 2007, 17, 1613.

[8] Tian, Z. Y.; Xie, S. Q.; Zhao, J.; Gao, W.-Y.; Wang, C. J. J. Henan Univ. 2008, 28, 469 (in Chinese). 
(田智勇, 谢松强, 赵瑾, 高文远, 王超杰, 河南大学学报, 2008, 28,469 .)

[9] Xiao, K. Y.; Dai, Y. Shi, W. Q.; Li, J. Z.; Li, Y.; Yin, S. F. Chin. J. Org. Chem. 2012, 32, 169 (in Chinese).

(肖克毅, 戴亚, 石万棋, 李剑忠, 李颖, 尹述凡, 有机化学, 2012, 32, 169.)

[10] Liu, W. Q.; Tao, L. M.; Zang, Z. L.; Li, Y. H.; Xu, X. H. Chin. J. Synth. Chem. 2009, 17, 64 (in Chinese).

(刘文奇, 陶李明，蔵中林，李银辉，许新华，合成化学，2009, 17, 64.)

[11] Qin, S. H.; Liu, A. Q.; Yu, S. H.; Wang, F. L. Chin. Pharmacol. Bull. 2013, 29, 145 (in Chinese)

(秦三海, 刘爱芹, 于胜海, 王风玲, 中国药理学通报, 2013, 29, 145.)

[12] Liu, Y. M.; Lee, H. Y.; Lai, M. J.; Pan, S. L.; Huang, H. L.; Kuo, F. C.; Chen, M. C.; Liou, J. P. Org. Biomol. Chem. 2015, 13, 10226.

[13] Kasprzak, A.; Koszytkowska-Stawińska, M.; Nowicka, A. M.; Buchowicz, W.; Poplawska, M. J. Org. Chem. 2019, 84, 15900.

[14] Rich, T. A.; Shepard, R. C.; Mosley, S. T. J. Clin. Oncol. 2004, 22, 2214

[15] Wu, L. Q.; Yang, X. J.; Peng, Q. J. Sun, G. F. Eur. J. Med. Chem. 2017, 127, 599

[16] Liu, W. Q.; Yin, X. H.; Zang, Z. L.; Yu, X. F.; Tao, L. M.; Li, Y. J. Xu, X. H. Chin. J. Org. Chem. 2009, 29, 2021 (in Chinese) (刘文奇, 尹显洪, 藏中林, 余晓芬, 陶李明, 李言杰, 许新华, 有机化学, 2009, 29, 2021.)

[17] He, W. B.; Gao, L. Q.; Chen, X. J.; Wu, Z. L.; Huang, Y.; Cao, Z.; Xu, X. H. He, W. -M. Chin. Chem. Lett. 2020, 31, 1895.

[18] Wu, Y.; Lin, Y.-W.; He, W.-M. Chin. Chem. Lett. 2020, 31, 2999.
[19] Wu, C.; Xiao, H. J.; Wang, S. W.; Tang, M. S.; Tang, Z. L.; Xia, W.; Li, W. F.; Cao, Z.; He, W. -M. ACS Sustainable Chem. Eng. 2019, 7,2169

[20] Madhunapantula, S. V.; Desai, D.; Sharma, A.; Huh, S. J.; Amin, S.; Robertson, G. P. Mol. Cancer Ther. 2008, 7, 1297.

[21] Sun, C. L.; Sun, X. N.; Pu, Y. X.; Li, C. Y.; Sun, L. J.; Wang, J.; Li, Y. Z. Chin. J. Org. Chem. 2017, 37, 440 (in Chinese). (孙成路, 孙晓娜, 蒲雨昕, 李传银, 孙丽杰, 王静, 李益政, 有 机化学, 2017, 37, 440.)

[22] Wang, L. X.; Li, N. B.; Wang, H. J.; Liu, W.; Diao, H. P.; Xu, X. H. Chin. J. Org. Chem. 2019, 39, 1802 (in Chinese). (王灵晓，李宁波，王浩江，刘文，フ海鹏，许新华，有机化学, 2019, 39, 1802.)

[23] Chen, J.-Y.; Zhong, C.-T.; Gui, Q.-W.; Zhou, Y.-M.; Fang, Y.-Y.; Liu, K.-J.; Lin, Y.-W.; Cao, Z.; He, W.-M. Chin. Chem. Lett. 2021, 32,475

[24] Zang, Z. L.; Liu, S. Q.; Chen, X.; Li, Y. J.; Zhou, B.; Xu, X. H. Acta Pham. Sin. 2006, 41, 1184 (in Chinese)

(藏中林, 刘少琼, 陈雄, 李言杰, 周冰, 许新华, 药学学报, 2006, 41, 1184.)

[25] Deng, X. H.; Xia, X.; Shao, L. L.; Zang, Z. L.; Li, Y. J.; Xu, X. H. Chin. J. Synth. Chem. 2008, 16, 285 (in Chinese).

(邓新华, 夏湘, 邵玲玲, 藏中林, 李言杰, 许新华, 合成化学, 2008, 16, 282.)

[26] Wang, L. X.; Qiao, J.; Wei, J. C.; Liang, Z. W.; Xu, X. H.; Li, N. B. Tetrahedron 2020, 76, 130750

[27] Yu, A. H.; Xu, X. H. Chem. Reagents 2020, 42,1370 (in Chinese). (喻爱和，许新华，化学试剂, 2020, 42, 1370.) 